Pacific

Journal of

Mathematics

\title{
COMPARISON OF COMPACT INDUCTION WITH PARABOLIC INDUCTION
}

Guy Henniart and Marie-France Vigneras 


\title{
COMPARISON OF COMPACT INDUCTION WITH PARABOLIC INDUCTION
}

\author{
Guy Henniart and Marie-France Vigneras
}

\begin{abstract}
In memory of Jon Rogawski
I (GH) have very fond memories of an extraordinary expedition to the Grand Canyon with Jon. I have in mind these happy remembrances, and others of a more mathematical kind, in dedicating the present paper to him. I (MFV) first met Jon in Paris when he was a student visiting École Normale Supérieure; at that time, he was already gifted, charming, fluent in French, and full of music and mathematics. During another visit that Jon made to ENS, we discussed in detail his preprint on the Jacquet-Langlands local correspondence for division algebras; my collaboration with Bernstein, Deligne and Kazhdan emerged from this. Afterwards, I always looked forward to seeing him at scientific meetings, and I was enriched by being welcomed by his wife Julie and their four children to their home in Los Angeles.
\end{abstract}

We and our French colleagues all share a sense of great loss.

MSC2010: primary 22E50; secondary 11F70.

Keywords: representations modulo $p$ of reductive $p$-adic groups, compact induction, parabolic induction, Satake isomorphism. 
Let $F$ be a nonarchimedean locally compact field of residual characteristic $p$, let $G$ be a connected reductive $F$-group, and let $K$ be a special parahoric subgroup of $G(F)$. We choose a parabolic $F$-subgroup $P$ of $G$ with Levi decomposition $P=M N$ in good position with respect to $K$. Let $C$ be an algebraically closed field of characteristic $p$, and $V$ an irreducible smooth $C$-representation of $K$. We investigate the natural intertwiner from the compact induced representation c-Ind ${ }_{K}^{G(F)} V$ to the parabolic induced representation $\operatorname{Ind}_{P(F)}^{G(F)}\left(\right.$ c-Ind $\left.M_{M(F) \cap K}^{M(F)} V_{N(F) \cap K}\right)$. Under a regularity condition on $V$, we show that the intertwiner becomes an isomorphism after localization at a specific Hecke operator. When $F$ has characteristic $0, G$ is $F$-split and $K$ is hyperspecial, the result was essentially proved by Herzig. We define the notion of $\boldsymbol{K}$-supersingularity for an irreducible smooth $\boldsymbol{C}$-representation of $G(F)$ which extends Herzig's definition for admissible irreducible representations and we give a list of irreducible representations which are neither supercuspidal nor $\boldsymbol{K}$-supersingular. 
1. Introduction 458

2. Generalities on the Satake homomorphisms 461

3. Representations of $G(k) \quad 468$

4. Representations of $G(F) \quad 472$

5. Hecke operators 478

6. Proof of the main theorem 485

7. Supersingular representations of $G(F) \quad 488$

Acknowledgements $\quad 494$

References $\quad 494$

\section{Introduction}

Let $F$ be a nonarchimedean locally compact field of residual characteristic $p$, let $G$ be a reductive connected $F$-group, and let $C$ be an algebraically closed field of characteristic $p$. We are interested in smooth admissible $C$-representations of $G(F)$. Two induction techniques are available: compact induction c-Ind ${ }_{F}^{G(F)}$ from a compact open subgroup $K$ of $G(F)$ and parabolic induction $\operatorname{Ind}_{P(F)}^{G(F)}$ from a parabolic subgroup $P(F)$ with Levi decomposition $P(F)=M(F) N(F)$. Here we want to investigate the interaction between the two inductions.

More specifically, assume that $G(F)=P(F) K$ and

$$
P(F) \cap K=(M(F) \cap K)(N(F) \cap K) .
$$

We construct (Definition 2.1), for any finite-dimensional smooth $C$-representation $V$ of $K$, a canonical intertwiner

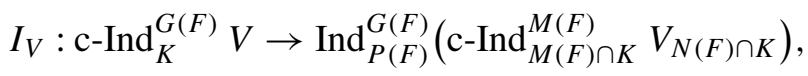

where $V_{N(F) \cap K}$ stands for the $N(F) \cap K$-coinvariants in $V$, and a canonical algebra homomorphism

$$
\mathscr{S}^{\prime}: \mathscr{H}(G(F), K, V) \rightarrow \mathscr{H}\left(M(F), M(F) \cap K, V_{N(F) \cap K}\right),
$$

where, as in [Henniart and Vigneras 2011], the Hecke algebra $\mathscr{H}(G(F), K, V)$ is $\operatorname{End}_{G(F)} \mathrm{c}-\operatorname{Ind}_{K}^{G(F)} V$ seen as an algebra of double cosets of $K$ in $G$, and similarly for

$$
\mathscr{H}\left(M(F), M(F) \cap K, V_{N(F) \cap K}\right) .
$$

By construction,

$$
\left(I_{V}(\Phi(f))\right)(g)=\mathscr{Y}^{\prime}(\Phi)\left(I_{V}(f)(g)\right),
$$

for $f \in \mathrm{c}-\operatorname{Ind}_{K}^{G(F)} V, \Phi \in \mathscr{H}(G(F), K, V), g \in G(F)$. 
Let $V^{*}$ be the contragredient representation of $V$. We constructed in [Henniart and Vigneras 2011] a Satake homomorphism

$$
\mathscr{S}: \mathscr{H}\left(G(F), K, V^{*}\right) \rightarrow \mathscr{H}\left(M(F), M(F) \cap K,\left(V^{*}\right)^{N(F) \cap K}\right) .
$$

Here we show that $\mathscr{S}^{\prime}$ and $\mathscr{Y}$ are related by a natural anti-isomorphism of Hecke algebras (Proposition 2.3).

We study $I_{V}$ further in the particular case where $K$ is a special parahoric subgroup and $V$ is irreducible. Such a $V$ is trivial on the pro- $p$-radical $K_{+}$of $K$. The quotient $K / K_{+}$is the group of $k$-points of a connected reductive $k$-group $G_{k}$, so that we can use the theory of finite reductive groups in natural characteristic. We write $K / K_{+}=G(k)$. The image of $P(F) \cap K=P_{0}$ in $G(k)$ is the group of $k$-points of a parabolic subgroup of $G_{k}$. We write $P_{0} / P_{0} \cap K_{+}=P(k)$, and we use similar notations for $M$ and $N$, for the opposite parabolic subgroup $\bar{P}=M \bar{N}$ (Section 4A), and for a minimal parabolic $F$-subgroup $\bar{B}$ of $G$ contained in $\bar{P}$, of Levi decomposition $\bar{B}=Z \bar{U}$.

We say that $V$ is $\bar{P}$-regular when the stabilizer $\bar{P}_{V}(k)$ in $G(k)$ of the line $V^{\bar{U}(k)}$ is contained in $\bar{P}(k)$ (this does not depend on the choice of $\bar{B}$ ). An equivalent definition is that, for $h \in K$ which does not belong to $P_{0} \bar{P}_{0}$, the kernel of the quotient map $V \rightarrow V_{N(k)}$ contains $h V^{\bar{N}(k)}$ (Definition 3.6 and Corollary 3.19).

We choose a maximal $F$-split torus $S$ in $M$ such that $K$ stabilizes a special vertex in the apartment of $G(F)$ associated to $S$. We choose an element $s \in S(F)$ which is central in $M(F)$ and strictly $N$-positive, in the sense that conjugation by $s$ strictly contracts the compact subgroups of $N(F)$. There is a unique Hecke operator $T_{M}$ in $\mathscr{H}\left(M(F), M_{0}, V_{N(k)}\right)$ with support in $M_{0} s$ and value at $s$ the identity morphism of $V_{N(k)}$. We prove (Proposition 4.5):

Proposition 1.1. The map $\mathscr{S}^{\prime}$ is a localization at $T_{M}$.

This means that $\mathscr{Y}^{\prime}$ is injective, that $T_{M}$ belongs to the image of $\mathscr{Y}^{\prime}$ and is central and invertible in $\mathscr{H}\left(M(F), M_{0}, V_{N(k)}\right)$, and that

$$
\mathscr{H}\left(M(F), M_{0}, V_{N(k)}\right)=\mathscr{S}^{\prime}(\mathscr{H}(G(F), K, V))\left[T_{M}^{-1}\right] .
$$

This is a consequence of the analogous property of $\mathscr{Y}$ proved in [Henniart and Vigneras 2011].

In this particular case, following a suggestion of Abe, we show that $I_{V}$ is injective. We introduce the localization $\Theta$ of $I_{V}$ at $T_{M}$. As $I_{V}$ is injective, its localization $\Theta$ is injective. Our main theorem is this:

Theorem 1.2 (Theorem 4.6). The map

$\Theta: \mathscr{H}\left(M(F), M_{0}, V_{N(k)}\right) \otimes \mathscr{H}(G(F), K, V), \varphi^{\prime} \mathrm{c}-\operatorname{Ind}_{K}^{G(F)} V \rightarrow \operatorname{Ind}_{P(F)}^{G(F)}\left(\mathrm{c}-\operatorname{Ind}_{M(F) \cap K}^{M(F)} V_{N(k)}\right)$

is bijective if $V$ is $\bar{P}$-regular. 
The special case when $F$ has characteristic $0, G$ is $F$-split, and $K$ is hyperspecial was proved in [Herzig 2011] (see also [Abe 2011]). In this case the Hecke algebras are commutative.

Writing $\mathscr{L}_{G}(V)$ for the center of $\mathscr{H}(G(F), K, V)$ and $\mathscr{L}_{M}\left(V_{N(k)}\right)$ for the center of $\mathscr{H}\left(M(F), M(F) \cap K, V_{N(k)}\right)$, the theorem implies by specialization:

Corollary 1.3. If $V$ is $\bar{P}$-regular, for any right $\mathscr{L}_{M}\left(V_{N(k)}\right)$-module $\chi$, the representations of $G(F)$

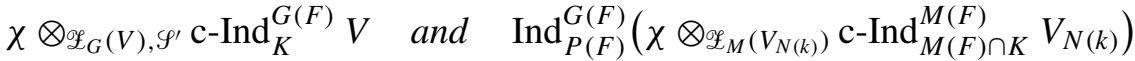

are isomorphic.

To prove the theorem, we follow the method of Herzig and decompose $I_{V}$ as the composite $I_{V}=\zeta \circ \xi$ of two $G(F)$-equivariant maps: the natural inclusion $\xi$ of c-Ind $K_{K}^{G(F)} V$ in c-Ind $_{K}^{G(F)}\left(\mathrm{c}-\operatorname{Ind}_{P(k)}^{G(k)} V_{N(k)}\right)$ and the natural map

$$
\zeta: \mathrm{c}-\operatorname{Ind}_{K}^{G(F)}\left(\mathrm{c}-\operatorname{Ind}_{P(k)}^{G(k)} V_{N(k)}\right) \rightarrow \operatorname{Ind}_{P(F)}^{G(F)}\left(\mathrm{c}-\operatorname{Ind}_{M(F) \cap K}^{M(F)} V_{N(k)}\right),
$$

associated to the quotient map c-Ind ${ }_{P(k)}^{G(k)} V_{N(k)} \rightarrow V_{N(k)}$ (see (2) below). We write $\mathscr{P}$ for the parahoric subgroup inverse image of $P(k)$ in $K$ and $T_{\mathscr{P}}$ for the Hecke operator in $\mathscr{H}\left(G(F), \mathscr{P}, V_{N(k)}\right)$ with support $\mathscr{P} s \mathscr{P}$ and value at $s$ the identity of $V_{N(k)}$. With no regularity assumption on $V$, we prove

$$
\zeta \circ T_{\mathscr{P}}=T_{M} \circ \zeta .
$$

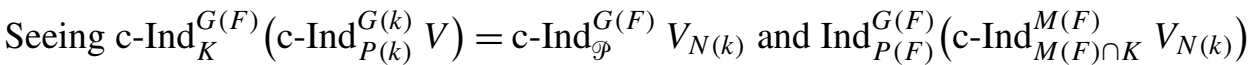
as $C[T]$-modules via $T_{\mathscr{P}}$ and $T_{M}$, the map $\zeta$ is $C[T]$-linear, and using Corollary 6.5 we prove:

Theorem 1.4. The localization at $T$ of $\zeta$ is an isomorphism.

To study $\xi$, we consider the Hecke operator $T_{G}$ in $\mathscr{H}(G(F), K, V)$ with support $K s K$ and value at $s$ the natural projector $V \rightarrow V^{\bar{N}(k)}$, and the Hecke operator $T_{K, \Phi}$ from c-Ind $\mathrm{I}_{\mathscr{P}}^{G(F)} V_{N(k)}$ to c-Ind ${ }_{K}^{G(F)} V$ with support $K s \mathscr{P}$ and value at $s$ given by the natural isomorphism $V_{N(k)} \rightarrow V^{\bar{N}(k)}$. With no regularity assumption on $V$, we prove

$$
T_{K, \mathscr{P}} \circ \xi=T_{G} .
$$

Assuming that $V$ is $\bar{P}$-regular, we prove

$$
\begin{aligned}
\xi \circ T_{K, \mathscr{P}} & =T_{\mathscr{P}}, \\
\mathscr{S}^{\prime}\left(T_{G}\right) & =T_{M} .
\end{aligned}
$$

Seeing c-Ind ${ }_{K}^{G(F)} V$ as a $C[T]$-module via $T_{G}=\left(\mathscr{S}^{\prime}\right)^{-1}\left(T_{M}\right)$, the map $\xi$ is $C[T]$ linear and: 
Theorem 1.5. The localization at $T$ of $\xi$ is an isomorphism when $V$ is $\bar{P}$-regular.

These two theorems imply that $\Theta$ is an isomorphism when $V$ is $\bar{P}$-regular.

Following Herzig and Abe, we define the notion of $K$-supersingularity.

Definition 1.6. We say that an irreducible smooth $C$-representation $\pi$ of $G(F)$ is $K$-supersingular when

$$
\mathscr{H}\left(M(F), M_{0}, V_{N(k)}\right) \otimes \mathscr{H}(G(F), K, V), \mathscr{S}^{\prime} \operatorname{Hom}_{G(F)}\left(\mathrm{c}-\operatorname{Ind}_{K}^{G(F)} V, \pi\right)=0
$$

for any irreducible smooth $C$-representation $V$ of $K$ and any standard Levi subgroup $M \neq G$.

If $\pi$ is a smooth irreducible $C$-representation of $G(F)$, we say that $\pi$ is supercuspidal if $\pi$ is not a subquotient of a proper parabolically induced representation $\operatorname{Ind}_{P(F)}^{G(F)} \tau, P \neq G$, from an irreducible smooth $C$-representation $\tau$. Note that for an admissible $\pi$, our requirement for supercuspidality is stronger than the one used in [Herzig 2011, Definition 9.12]: he only asks that $\pi$ not be a subquotient of a proper parabolically induced representation from an irreducible admissible $C$-representation. In their context and with Herzig's definition, Herzig and Abe [Abe 2011, Corollary 5.10] show that, for admissible $\pi, K$-supersingularity is equivalent to supercuspidality. We expect that the same is true, for admissible $\pi$, in our more general context and with our definition. Here are the partial results we have in that direction:

Theorem 1.7. Let $\pi$ be an irreducible smooth $C$-representation of $G(F)$.

i. If $\pi$ is isomorphic to a subrepresentation or is an admissible quotient of $\operatorname{Ind}_{P(F)}^{G(F)} \tau$ as above, then $\pi$ is not $K$-supersingular.

ii. If $\pi$ is admissible and

$$
\mathscr{H}\left(M(F), M_{0}, V_{N(k)}\right) \otimes \mathscr{H}(G(F), K, V), \mathscr{S}^{\prime} \operatorname{Hom}_{G(F)}\left(\mathrm{c}-\operatorname{Ind}_{K}^{G(F)} V, \pi\right) \neq 0
$$

for some $\bar{Q}$-regular irreducible subrepresentation $V$ of $\left.\pi\right|_{K}$ and some standard parabolic subgroups $P=M N \subset Q=L N^{\prime} \neq G$, then $\pi$ is a quotient of $\operatorname{Ind}_{Q(F)}^{G(F)} \tau$ for an admissible irreducible smooth $C$-representation $\tau$ of $L(F)$.

\section{Generalities on the Satake homomorphisms}

In this chapter we give a functorial construction of Herzig's Satake transform $\mathscr{Y}^{\prime}$ in a rather general situation. Let $C$ be a field, $G$ a locally profinite group, $K$ an open subgroup of $G$, and $P$ a closed subgroup of $G$ satisfying the "Iwasawa decomposition" $G=K P$. We choose a smooth $C[K]$-module $V$. As in [Henniart and Vigneras 2011], we assume that $P$ is the semidirect product of a closed normal subgroup $N$ and of a closed subgroup $M$, and that $K \cap P$ is the semidirect product of $N \cap K$ by $M \cap K$. We also impose: 
A1) Each double coset $K g K$ in $G$ is the union of a finite number of cosets $K g^{\prime}$ and the union of a finite number of cosets $g^{\prime \prime} K$ (the first condition for $g$ is equivalent to the second for $g^{-1}$ ).

A2) $V$ is a finite-dimensional $C$-vector space.

The smooth $C[K]$-module $V$ gives rise to a compactly induced representation c-Ind $K_{K}^{G} V$ and a smooth $C[P]$-module $W$ gives rise to the full smooth induced representation $\operatorname{Ind}_{P}^{G} W$. We consider the space of intertwiners

$$
\mathscr{F}:=\operatorname{Hom}_{G}\left(\mathrm{c}-\operatorname{Ind}_{K}^{G} V, \operatorname{Ind}_{P}^{G} W\right) .
$$

By Frobenius reciprocity for compact induction (as $K$ is open in $G$ ), the $C$-module $\mathscr{F}$ is canonically isomorphic to $\operatorname{Hom}_{K}\left(V, \operatorname{Res}_{K}^{G} \operatorname{Ind}_{P}^{G} W\right)$; to an intertwiner $I$ we associate the function $v \mapsto I[1, v]_{K}$, where $[1, v]_{K}$ is the function in $\operatorname{cond}_{K}^{G} V$ with support $K$ and value $v$ at 1 . By the Iwasawa decomposition and the hypothesis that $K$ is open in $G$, we get by restricting functions to $K$ an isomorphism of $C[K]$-modules from $\operatorname{Res}_{K}^{G} \operatorname{Ind}_{P}^{G} W$ onto $\operatorname{Ind}_{P \cap K}^{K}\left(\operatorname{Res}_{P \cap K}^{P} W\right)$. Using now Frobenius reciprocity for the full smooth induction $\operatorname{Ind}_{P \cap K}^{K}$ from $P \cap K$ to $K$, we finally get a canonical $C$-linear isomorphism

$$
\mathscr{g} \simeq \operatorname{Hom}_{P \cap K}(V, W)
$$

(we now omit mentioning the obvious restriction functors in the notation); this map associates to an intertwiner $I$ the function $v \mapsto\left(I[1, v]_{K}\right)(1)$.

We could have proceeded differently, first applying Frobenius reciprocity to $\operatorname{Ind}_{P}^{G} W$, getting $\mathscr{\mathscr { F }} \simeq \operatorname{Hom}_{P}\left(\mathrm{c}-\operatorname{Ind}_{K}^{G} V, W\right)$, then identifying $\operatorname{Res}_{P}^{G} \mathrm{c}-\operatorname{Ind}_{K}^{G} V$ with c-Ind ${ }_{P \cap K}^{P} V$, and finally applying Frobenius reciprocity to $\mathrm{c}-\operatorname{Ind}_{P \cap K}^{P} V$. In this way we also obtain an isomorphism of $\mathscr{g}$ onto $\operatorname{Hom}_{P \cap K}(V, W)$, which is readily checked to be the same as the preceding one.

Assume also that $W$ is a smooth $C[M]$-module, seen as a smooth $C[P]$-module by inflation. Then $\operatorname{Ind}_{P}^{G} W$ is the parabolic induction of $W$, and $\operatorname{Hom}_{P \cap K}(V, W)$ identifies with $\operatorname{Hom}_{M \cap K}\left(V_{N \cap K}, W\right)$, where $V_{N \cap K}$ is the space of coinvariants of $N \cap K$ in $V$. With that identification, an intertwiner $I$ is sent to the map from $V_{N \cap K}$ to $W$ sending the image $\bar{v}$ of $v \in V$ in $V_{N \cap K}$ to $\left(I[1, v]_{K}\right)(1)$. By Frobenius reciprocity again, $\operatorname{Hom}_{M \cap K}\left(V_{N \cap K}, W\right)$ is isomorphic to $\operatorname{Hom}_{M}\left(\operatorname{cc}^{-\operatorname{Ind}_{M \cap K}} V_{N \cap K}, W\right)$, so overall we obtain an isomorphism

$$
j: \mathscr{g}=\operatorname{Hom}_{G}\left(\mathrm{c}-\operatorname{Ind}_{K}^{G} V, \operatorname{Ind}_{P}^{G} W\right) \rightarrow \operatorname{Hom}_{M}\left(\mathrm{c}-\operatorname{Ind}_{M \cap K}^{M} V_{N \cap K}, W\right),
$$

which associates to $I \in \mathscr{E}$ the $C[M]$-linear map sending $[1, \bar{v}]_{M \cap K}$ to $\left(I[1, v]_{K}\right)(1)$. The reciprocal isomorphism sends $I^{\prime} \in \operatorname{Hom}_{M}\left(\mathrm{c}-\operatorname{Ind}_{M \cap K}^{M} V_{N \cap K}, W\right)$ to the element in $\operatorname{Hom}_{G}\left(\mathrm{c}-\operatorname{Ind}_{K}^{G} V, \operatorname{Ind}_{P}^{G} W\right)$ which, for $v \in V$, sends $[1, v]_{K}$ to the unique function with value $I^{\prime}\left([1, \overline{k v}]_{M \cap K}\right)$ at $k \in K$. 
For $W=\mathrm{c}-\operatorname{Ind}_{M \cap K}^{M} V_{N \cap K}$, the isomorphism $j$ is written $j_{V}$ : $j_{V}: \operatorname{Hom}_{G}\left(\mathrm{c}-\operatorname{Ind}_{K}^{G} V, \operatorname{Ind}_{P}^{G}\left(\mathrm{c}-\operatorname{Ind}_{M \cap K}^{M} V_{N \cap K}\right)\right) \rightarrow \operatorname{End}_{M}\left(\mathrm{c}-\operatorname{Ind}_{M \cap K}^{M} V_{N \cap K}\right)$.

Definition 2.1. We define $I_{V}$ in $\operatorname{Hom}_{G}\left(\mathrm{c}-\operatorname{Ind}_{K}^{G} V, \operatorname{Ind}_{P}^{G}\left(\mathrm{c}-\operatorname{Ind}_{M \cap K}^{M} V_{N \cap K}\right)\right)$ such that $j_{V}\left(I_{V}\right)$ is the unit element of $\operatorname{End}_{M}\left(\mathrm{c}-\operatorname{Ind}_{M \cap K}^{M} V_{N \cap K}\right)$. The intertwiner $I_{V}$ is determined by the condition

$$
\left(I_{V}[1, v]_{K}\right)(1)=[1, \bar{v}]_{M \cap K}
$$

for all $v \in V$.

The isomorphism $j$ is natural in $V$ and $W$. The functor

$$
\mathscr{F}_{V}: W \mapsto \operatorname{Hom}_{G}\left(\mathrm{c}-\operatorname{Ind}_{K}^{G} V, \operatorname{Ind}_{P}^{G} W\right)
$$

from the category of smooth $C[M]$-modules to the category of sets is representable by c-Ind ${ }_{M \cap K}^{M} V_{N \cap K}$. Let now $V^{\prime}$ be another finite-dimensional smooth $C[K]$ module. Any $G$-intertwiner

$$
b: \mathrm{c}-\operatorname{Ind}_{K}^{G} V \rightarrow \mathrm{c}-\operatorname{Ind}_{K}^{G} V^{\prime}
$$

gives a morphism of functors $\mathscr{F}_{V^{\prime}} \rightarrow \mathscr{F}_{V}$. By the representability of $\mathscr{F}_{V}$ and $\mathscr{F}_{V}$, there is then a unique $C[M]$-morphism

$$
\mathscr{S}^{\prime}(b): \mathrm{c}-\operatorname{Ind}_{M \cap K}^{M} V_{N \cap K} \rightarrow \mathrm{c}-\operatorname{Ind}_{M \cap K}^{M} V_{N \cap K}^{\prime}
$$

such that the diagram

$$
\begin{aligned}
& \operatorname{Hom}_{G}\left({\mathrm{c}-\operatorname{Ind}_{K}^{G}}^{\prime} V^{\prime}, \operatorname{Ind}_{P}^{G} W\right) \stackrel{j^{\prime}}{\longrightarrow} \operatorname{Hom}_{M}\left(\mathrm{c}-\operatorname{Ind}_{M \cap K}^{M} V_{N \cap K}^{\prime}, W\right) \\
& I^{\prime} \mapsto I^{\prime} \circ b \downarrow \quad I^{\prime} \mapsto I^{\prime} \circ \mathscr{S}^{\prime}(b) \downarrow \\
& \operatorname{Hom}_{G}\left(\mathrm{c}-\operatorname{Ind}_{K}^{G} V, \operatorname{Ind}_{P}^{G} W\right) \underset{j}{\longrightarrow} \operatorname{Hom}_{M}\left(\mathrm{c}-\operatorname{Ind}_{M \cap K}^{M} V_{N \cap K}, W\right)
\end{aligned}
$$

is commutative for all smooth $C[M]$-modules $W$ (the horizontal maps are the canonical isomorphisms constructed above, and the vertical maps are given by composition with $b$ or with $\mathscr{Y}^{\prime}(b)$ ). Taking $W=\mathrm{c}-\operatorname{Ind}_{M \cap K}^{M} V_{N \cap K}$, we get

$$
\mathscr{S}^{\prime}(b)=j\left(I_{V^{\prime}} \circ b\right),
$$

when $V_{N \cap K}=V_{N \cap K}^{\prime}$ as a representation of $M \cap K$. If $V^{\prime}$ is a third finite-dimensional smooth $C[K]$-module and

$$
b^{\prime}: \mathrm{c}-\operatorname{Ind}_{K}^{G} V^{\prime} \rightarrow \mathrm{c}-\operatorname{Ind}_{K}^{G} V^{\prime \prime}
$$


is a $G$-intertwiner, then $b^{\prime} \circ b: \mathrm{c}-\operatorname{Ind}_{K}^{G} V \rightarrow \mathrm{c}-\operatorname{Ind}_{K}^{G} V^{\prime \prime}$ is a $G$-intertwiner and we have obviously

$$
\mathscr{S}^{\prime}\left(b^{\prime} \circ b\right)=\mathscr{Y}^{\prime}\left(b^{\prime}\right) \circ \mathscr{Y}^{\prime}(b) .
$$

Taking $V=V^{\prime}=V^{\prime \prime}$, we get an algebra homomorphism

$$
\varphi^{\prime}: \operatorname{End}_{G}\left(\mathrm{c}-\operatorname{Ind}_{K}^{G} V\right) \rightarrow \operatorname{End}_{M}\left(\mathrm{c}-\operatorname{Ind}_{M \cap K}^{M} V_{N \cap K}\right)
$$

such that

$$
j(I \circ b)=j(I) \circ \mathscr{Y}^{\prime}(b)
$$

for $I$ in $\operatorname{Hom}_{G}\left(\mathrm{c}-\operatorname{Ind}_{K}^{G} V, \operatorname{Ind}_{P}^{G} W\right)$.

By the naturality of $j$ in $W$, for any homomorphism $\alpha: W^{\prime} \rightarrow W$ of smooth $C[M]$-modules, we have a commutative diagram

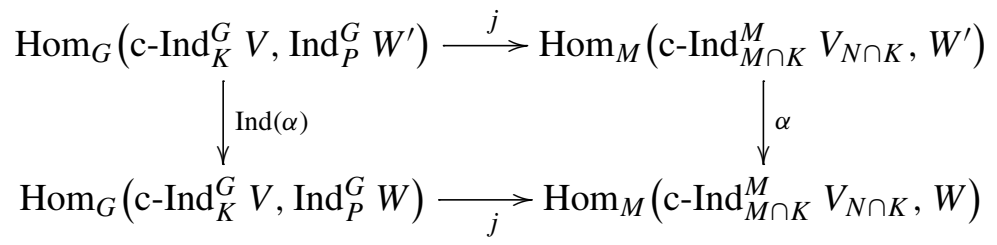

for any $V$. For $W=W^{\prime}$, we obtain $j\left(\left(\operatorname{Ind}_{P}^{G} \alpha\right) \circ I\right)=\alpha \circ j(I)$ for $\alpha \in \operatorname{End}_{M}(W)$. We have

$$
j\left(\left(\operatorname{Ind}_{P}^{G} \alpha\right) \circ I_{V}\right)=\alpha
$$

for all $\alpha$ in $\operatorname{Hom}_{M}\left(\mathrm{c}-\operatorname{Ind}_{M \cap K}^{M} V_{N \cap K}, W\right)$. For $W=W^{\prime}=\mathrm{c}-\operatorname{Ind}_{M \cap K}^{M} V_{N \cap K}$, we deduce

$$
I_{V} \circ b=\left(\operatorname{Ind}_{P}^{G} \varphi^{\prime}(b)\right) \circ I_{V}
$$

for $b \in \operatorname{End}_{G}\left(\mathrm{c}-\operatorname{Ind}_{K}^{G} V\right)$, by applying $j_{V}^{-1}$ to (5).

We now want to interpret the previous results in terms of actions of Hecke algebras. By Frobenius reciprocity, $\operatorname{Hom}_{G}\left(\mathrm{c}-\operatorname{Ind}_{K}^{G} V, \mathrm{c}-\operatorname{Ind}_{K}^{G} V^{\prime}\right)$ identifies with $\operatorname{Hom}_{K}\left(V, \operatorname{Res}_{K}^{G} \mathrm{c}-\operatorname{Ind}_{K}^{G} V^{\prime}\right)$, as a $C$-module; to a $G$-intertwiner $b$ we associate the map $v \mapsto b_{v}:=b\left([1, v]_{K}\right)$. From such a $b$, we get a map

$$
\Phi_{b}: G \rightarrow \operatorname{Hom}_{C}\left(V, V^{\prime}\right), \quad g \mapsto\left(v \mapsto b_{v}(g)\right) .
$$

Thus we identify $\operatorname{Hom}_{G}\left(\mathrm{c}-\operatorname{Ind}_{K}^{G} V, \mathrm{c}-\operatorname{Ind}_{K}^{G} V^{\prime}\right)$ with the space $\mathscr{H}\left(G, K, V, V^{\prime}\right)$ of functions $\Phi$ from $G$ to $\operatorname{Hom}_{C}\left(V, V^{\prime}\right)$ such that:

(i) $\Phi\left(k^{\prime} g k\right)=k^{\prime} \circ \Phi(g) \circ k$ for $k, k^{\prime}$ in $K, g$ in $G$, where we have written $k, k^{\prime}$ for the endomorphisms $v \mapsto k v, v^{\prime} \mapsto k^{\prime} v^{\prime}$ of $V$ and of $V^{\prime}$;

(ii) The support of $\Phi$ is a finite union of double cosets $K g K$. 
The natural map $\mathscr{H}\left(G, K, V, V^{\prime}\right) \times \mathrm{c}-\operatorname{Ind}_{K}^{G} V \rightarrow \mathrm{c}-\operatorname{Ind}_{K}^{G} V^{\prime}$ is given by convolution

$$
(\Phi * f)(g)=\sum_{h \in G / K} \Phi(h)\left(f\left(h^{-1} g\right)\right)=\sum_{h \in K \backslash G} \Phi\left(g h^{-1}\right)(f(h)) .
$$

The composition

$$
\mathscr{H}\left(G, K, V^{\prime}, V^{\prime \prime}\right) \times \mathscr{H}\left(G, K, V, V^{\prime}\right) \rightarrow \mathscr{H}\left(G, K, V, V^{\prime \prime}\right)
$$

corresponding to the composition of intertwiners is given by convolution

$$
(\Phi * \Psi)(g)=\sum_{h \in G / K} \Phi(h) \Psi\left(h^{-1} g\right)=\sum_{h \in K \backslash G} \Phi\left(g h^{-1}\right) \Psi(h)
$$

(the term $\Phi(h) \Psi\left(h^{-1} g\right)(v)$ vanishes, for fixed $g \in G$ and $v \in V$, outside finitely many cosets $K h$, so that the sum makes sense). The map

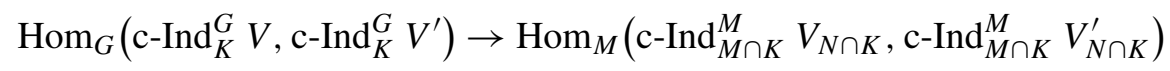

taking $b$ to $\mathscr{S}^{\prime}(b)$ translates into a map

$$
\mathscr{S}^{\prime}: \mathscr{H}\left(G, K, V, V^{\prime}\right) \rightarrow \mathscr{H}\left(M, M \cap K, V_{N \cap K}, V_{N \cap K}^{\prime}\right) .
$$

The next proposition shows that our definition of $\mathscr{Y}^{\prime}$ is equivalent to Herzig's.

Proposition 2.2. The homomorphism

$$
\mathscr{S}^{\prime}: \mathscr{H}\left(G, K, V, V^{\prime}\right) \rightarrow \mathscr{H}\left(M, M \cap K, V_{N \cap K}, V_{N \cap K}^{\prime}\right)
$$

is given by

$$
\mathscr{S}^{\prime}(\Phi)(m)(\bar{v})=\sum_{n \in(N \cap K) \backslash N} \overline{\Phi(n m)(v)} \text { for } m \in M, v \in V,
$$

where bars indicate the image in $V_{N \cap K}$ of elements in $V$ and similarly for $V^{\prime}$.

Proof. Let $b \in \operatorname{Hom}_{G}\left(\mathrm{c}-\operatorname{Ind}_{K}^{G} V, \mathrm{c}-\operatorname{Ind}_{K}^{G} V^{\prime}\right)$ and $\Phi_{b} \in \mathscr{H}\left(G, K, V, V^{\prime}\right)$ corresponding to $b$. We have, by (5),

$$
\mathscr{S}^{\prime}\left(\Phi_{b}\right)=\Phi_{\varphi^{\prime}(b)}=\Phi_{j\left(I_{V^{\prime}} \circ b\right)} .
$$

For $g \in G, v \in V, m \in M$, we have $\Phi_{b}(g)(v)=b\left([1, v]_{K}\right)(g)$ in $V^{\prime}$ and

$$
\mathscr{S}^{\prime}\left(\Phi_{b}\right)(m)(\bar{v})=\left(j\left(I_{V^{\prime}} \circ b\right)\right)\left([1, \bar{v}]_{M \cap K}\right)(m)=\left(\left(I_{V^{\prime}} \circ b\right)\left([1, v]_{K}\right)(1)\right)(m)
$$

in $V_{N \cap K}^{\prime}$. Using the Iwasawa decomposition, we write in c-Ind ${ }_{K}^{G} V$

$$
b\left([1, v]_{K}\right)=\sum_{h} h^{-1}\left[1, \Phi_{b}(h)(v)\right]_{K}
$$

for $h$ running over a system of representatives of $(P \cap K) \backslash P$. 
We compute now the element $I_{V^{\prime}}\left(h^{-1}\left[1, \Phi_{b}(h)(v)\right]_{K}\right)(1)$ of c-Ind $\operatorname{In}_{M \cap K} V_{N \cap K}^{\prime}$. As $I_{V^{\prime}}$ is $G$-equivariant, we have in $\operatorname{Ind}_{P}^{G}\left(\mathrm{c}-\operatorname{Ind}_{M \cap K}^{M} V_{N \cap K}^{\prime}\right)$

$$
I_{V^{\prime}}\left(h^{-1}\left[1, \Phi_{b}(h)(v)\right]_{K}\right)=h^{-1} I_{V^{\prime}}\left(\left[1, \Phi_{b}(h)(v)\right]_{K}\right) .
$$

Taking the value at the unit element 1 of $G$, we obtain

$$
\begin{aligned}
\left(h^{-1} I_{V^{\prime}}\left(\left[1, \Phi_{b}(h)(v)\right]_{K}\right)\right)(1) & =I_{V^{\prime}}\left(\left[1, \Phi_{b}(h)(v)\right]_{K}\right)\left(h^{-1}\right) \\
& =h^{-1}\left(I_{V^{\prime}}\left(\left[1, \Phi_{b}(h)(v)\right]_{K}\right)(1)\right) .
\end{aligned}
$$

Recalling (3), this is equal to

$$
h^{-1}\left[1, \overline{\Phi_{b}(h)(v)}\right]_{M \cap K}=m_{h^{-1}}\left[1, \overline{\Phi_{b}(h)(v)}\right]_{M \cap K}=m_{h}^{-1}\left[1, \overline{\Phi_{b}(h)(v)}\right]_{M \cap K},
$$

where $m_{h}$ is the image of $h$ in $M$. We deduce

$$
\left(I_{V^{\prime}} \circ b\right)\left([1, v]_{K}\right)(1)=\sum_{h} m_{h}^{-1}\left[1, \overline{\Phi_{b}(h)(v)}\right]_{M \cap K} .
$$

For $m$ in a system of representatives of $(M \cap K) \backslash M$, and $n$ in a system of representatives of $(N \cap K) \backslash N$, the elements $\mathrm{nm}$ form a system of representatives of $(P \cap K) \backslash P$. We obtain

$$
\begin{aligned}
\left(I_{V^{\prime}} \circ b\right)\left([1, v]_{K}\right)(1) & =\sum_{m \in(M \cap K) \backslash M} m^{-1}\left[1, w_{m}\right]_{M \cap K}, \\
w_{m} & :=\sum_{n \in(N \cap K) \backslash N} \overline{\Phi_{b}(n m)(v) .}
\end{aligned}
$$

In [Henniart and Vigneras 2011] we constructed a Satake homomorphism

$$
\begin{aligned}
\mathscr{S}: \mathscr{H}\left(G, K, V, V^{\prime}\right) & \rightarrow \mathscr{H}\left(M, M \cap K, V^{N \cap K}, V^{\prime N \cap K}\right), \\
\mathscr{S}(\Phi)(m)(v) & =\sum_{n \in N /(N \cap K)} \Phi(m n)(v),
\end{aligned}
$$

for $v \in V^{N \cap K}$. To compare $\mathscr{S}^{\prime}$ with $\mathscr{S}$ we need to take the dual. Remark that $K$ acts on the dual space $V^{*}=\operatorname{Hom}_{C}(V, C)$ of $V$ via the contragredient representation, and that the dual of $V^{*}$ is isomorphic to $V$ by our finiteness hypothesis on $V$. It is straightforward to verify that the map

$$
\iota: \mathscr{H}\left(G, K, V^{\prime *}, V^{*}\right) \rightarrow \mathscr{H}\left(G, K, V, V^{\prime}\right), \quad \iota(\Phi)(g):=\left(\Phi\left(g^{-1}\right)\right)^{t},
$$

where the upper index $t$ indicates the transpose, is a $C$-isomorphism, and satisfies

$$
\iota(\Phi * \Psi)=\iota(\Psi) * \iota(\Phi)
$$

for $\Phi \in \mathscr{H}\left(G, K, V^{* *}, V^{*}\right), \Psi \in \mathscr{H}\left(G, K, V^{\prime *}, V^{\prime *}\right)$. 
The linear forms on $V$ which are $(N \cap K)$-fixed identify with the linear forms on $V_{N \cap K}$,

$$
\left(V_{N \cap K}\right)^{*} \simeq\left(V^{*}\right)^{N \cap K},
$$

and similarly for $V^{\prime}$ and $V^{\prime \prime}$. This leads to a natural $C$-linear isomorphism

$$
\iota_{M}: \mathscr{H}\left(M, M \cap K,\left(V^{*}\right)^{N \cap K},\left(V^{*}\right)^{N \cap K}\right) \rightarrow \mathscr{H}\left(M, M \cap K, V_{N \cap K}, V_{N \cap K}^{\prime}\right) .
$$

The following proposition describes the relation between the Satake homomorphisms $\mathscr{S}$ attached to $V^{\prime *}, V^{*}$ and $\mathscr{S}^{\prime}$ attached to $V, V^{\prime}$.

Proposition 2.3. The following diagram is commutative:

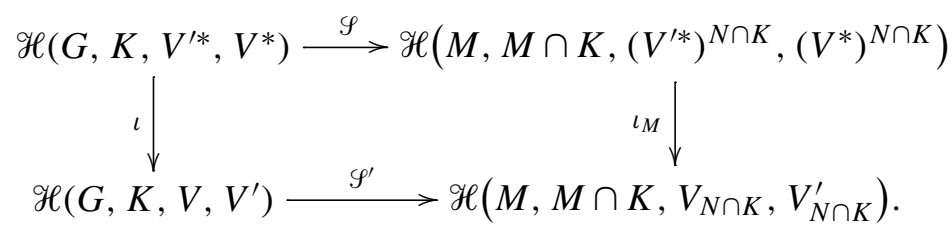

Proof. For $\Phi \in \mathscr{H}\left(G, K, V^{*}, V^{*}\right), m \in M$ and $v \in V$ of image $\bar{v}$ in $V_{N \cap K}$, we have:

$$
\begin{aligned}
\left(\left(\iota_{M} \circ \mathscr{S}\right) \Phi\right)(m)(\bar{v}) & =\left(\mathscr{S}(\Phi)\left(m^{-1}\right)\right)^{t}(\bar{v}) \\
& =\sum_{n \in N /(N \cap K)} \overline{\left(\Phi\left(m^{-1} n\right)\right)^{t}(v)} \\
& =\sum_{n \in(N \cap K) \backslash N} \overline{\left(\Phi\left((n m)^{-1}\right)\right)^{t}(v)} \\
& =\sum_{n \in(N \cap K) \backslash N} \overline{\iota(\Phi)(n m)(v)}=\left(\left(\mathscr{S}^{\prime} \circ \iota\right) \Phi\right)(m)(\bar{v}) .
\end{aligned}
$$

By this proposition, the Satake map $\mathscr{Y}$ is injective if and only if the map $\mathscr{Y}^{\prime}$ is injective because the maps $\iota$ and $\iota_{M}$ are isomorphisms.

Proposition 2.4. Let $V$ be a finite-dimensional smooth $C$-representation of $K$. If the homomorphisms $\mathscr{S}^{\prime}: \mathscr{H}\left(G, K, V^{\prime}, V\right) \rightarrow \mathscr{H}\left(M, M \cap K, V_{N \cap K}^{\prime}, V_{N \cap K}\right)$ are injective for all irreducible $C$-smooth representations $V^{\prime}$ of $K$, then the intertwiner

$$
I_{V}: \mathrm{c}-\operatorname{Ind}_{K}^{G} V \rightarrow \operatorname{Ind}_{P}^{G}\left(\mathrm{c}-\operatorname{Ind}_{M \cap K}^{M} V_{N \cap K}\right)
$$

is injective.

Proof. Assume that $I_{V}$ is not injective. Then the kernel of $I_{V}$ is a nonzero subrepresentation of $\mathrm{c}-\operatorname{Ind}_{K}^{G} V$, and contains an irreducible smooth $C[K]$-representation $V^{\prime}$. By Frobenius reciprocity, we get a nonzero intertwiner

$$
b \in \operatorname{Hom}_{G}\left(\mathrm{c}-\operatorname{Ind}_{K}^{G} V^{\prime}, \mathrm{c}-\operatorname{Ind}_{K}^{G} V\right)
$$


such that $I_{V} \circ b=0$. By assumption, the map

$$
\mathscr{S}^{\prime}: \mathscr{H}\left(G, K, V^{\prime}, V\right) \rightarrow \mathscr{H}\left(M, M \cap K, V_{N \cap K}^{\prime}, V_{N \cap K}\right)
$$

is injective. By the relation (5), this means that the map

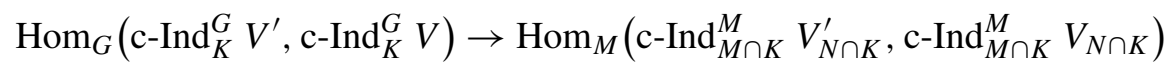

taking $b$ to $j\left(I_{V} \circ b\right)$ is injective, which gives a contradiction.

This criterion for the injectivity of $I_{V}$ was communicated to us by Noriyuki Abe.

\section{Representations of $G(k)$}

Let $C$ be an algebraically closed field of positive characteristic $p$, let $k$ be a finite field of the same characteristic $p$ and of cardinality $q$, and let $G$ be a connected reductive group over $k$. We fix a minimal parabolic $k$-subgroup $B$ of $G$ with unipotent radical $U$ and maximal $k$-subtorus $T$. Let $S$ be the maximal $k$-split subtorus of $T$, let $W=W_{G}=W(S, G)$ be the Weyl group, let $\Phi=\Phi_{G}$ be the roots of $S$ with respect to $U$ (called positive), and let $\Delta \subset \Phi$ be the subset of simple roots. For $a \in \Phi$, let $U_{a}$ be the unipotent subgroup denoted in [Bruhat and Tits $1984,5.1$ ] by $U_{(a)}$. A parabolic $k$-subgroup $P$ of $G$ containing $B$ is called standard, and has a unique Levi decomposition $P=M N$ with Levi subgroup $M$ (called standard) containing $T$. The standard parabolic subgroup $P=M U=U M$ is determined by $M$. There exists a unique subset $\Delta_{M} \subset \Delta$ such that $M$ is generated by $T, U_{a}, U_{-a}$ for $a$ in the subset $\Phi_{M}$ of $\Phi$ generated by $\Delta_{M}$. This determines a bijection between the subsets of $\Delta$ and the standard parabolic $k$-subgroups of $G$.

Let $\bar{B}=T \bar{U}$ be the opposite of $B=T U$, and $\bar{P}=M \bar{N}$ the opposite of $P$. We have $\bar{B}=w_{0} B w_{0}^{-1}$, where $w_{0}=w_{0}^{-1}$ is the longest element of $W$. The roots of $S$ with respect to $\bar{U}$, that is, the positive roots for $\bar{U}$, are the negative roots for $U$. The simple roots for $\bar{U}$ are the roots $-a$ for $a \in \Delta$.

For $a \in \Delta$, let $G_{a, k} \subset G(k)$ be the subgroup generated by the unipotent subgroups $U_{a}(k)$ and $U_{-a}(k)$, and let $T_{a, k}:=G_{a, k} \cap T(k)$.

Definition 3.1. Let $\psi: T(k) \rightarrow C^{*}$ be a $C$-character of $T(k)$. We denote by

$$
\Delta_{\psi}:=\left\{a \in \Delta \mid \psi\left(T_{a, k}\right)=1\right\}
$$

the set of simple roots $a$ such that $\psi$ is trivial on $T_{a, k}$.

Example 3.2. $G=\mathrm{GL}(n)$ and $S$ is the diagonal group. Then $T=S$ and the groups $T_{a}$ for $a \in \Delta$ are the subgroups $T_{i} \subset T$ for $1 \leq i \leq n-1$, with coefficients $x_{i}=x_{i+1}^{-1}$ and $x_{j}=1$ otherwise. When $k=\mathbb{F}_{2}$ is the field with 2 elements, $T(k)$ is the trivial group. 
Let $V$ be an irreducible $C$-representation of $G(k)$. When $P=M N$ is a standard parabolic subgroup of $G$, we recall that the natural action of $M(k)$ on $V^{N(k)}$ is irreducible [Cabanes and Enguehard 2004, Theorem 6.12]. In particular, taking the Borel subgroup $B=T U$, the dimension of the vector space $V^{U(k)}$ is 1 and the group $T(k)$ acts on $V^{U(k)}$ by a character $\psi_{V}$.

Proposition 3.3. The stabilizer in $G(k)$ of the line $V^{U(k)}$ is $P_{V}(k)$, where $P_{V}=$ $M_{V} N_{V}$ is a standard parabolic subgroup of $G$ associated to a subset $\Delta_{V} \subset \Delta_{\psi_{V}}$.

Proof. The stabilizer of $V^{U(k)}$ contains $B(k)$, and hence is of the form $P_{V}(k)$ for a standard parabolic subgroup $P_{V}$ of $G$ associated to the set $\Delta_{V}$ of simple roots $a \in \Delta$ such that $U_{-a}(k)$ acts trivially on $V^{U(k)}$. When $U_{-a}(k)$ acts trivially on $V^{U(k)}$, so does $G_{a, k}$ by definition of this group, implying that $a$ belongs to $\Delta_{\psi_{V}}$, by definition of this set.

Corollary 3.4. The dimension of $V$ is 1 if and only if $P_{V}=G$.

Proof. If the dimension of $V$ is 1 , then $V=V^{U(k)}$ and $P_{V}=G$. Conversely, if $P_{V}=G$, the line $V^{U(k)}$ is stable by $G(k)$, and hence is equal to $V$ because $V$ is irreducible.

Corollary 3.5. When $P=M N$ is a standard parabolic subgroup of $G$, the dimension of $V^{N(k)}$ is equal to 1 if and only if $P \subset P_{V}$.

The group $P_{V}$ measures the irregularity of $V$. A 1-dimensional representation $V$ is as little regular as possible $\left(P_{V}=G\right)$, and in general $V$ is as regular as possible when $P_{V}=B$.

Definition 3.6. Let $P$ be any parabolic $k$-subgroup of $G$. We say that $V$ is $P$ regular when the stabilizer in $G(k)$ of the line $V^{U(k)}$ is contained in $P(k)$, where $U$ is the unipotent radical of a minimal parabolic $k$-subgroup of $G$ contained in $P$.

The definition depends only on $P$ and not on the choice of $U$. The reason is that for a parabolic $k$-subgroup $P^{\prime} \subset P$ of $G$ and $g \in G(k)$, we have $g P^{\prime} g^{-1} \subset P$ if and only if $g \in P(k)$. As in the proof of [Borel and Tits 1965, Proposition 4.4 a)], the inclusion $P^{\prime} \subset g^{-1} P g \cap P$ implies $g^{-1} P g=P$, and $g \in P(k)$ because $P$ is equal to its own normalizer and is conjugate to a unique $k$-subgroup containing $P^{\prime}$.

We recall the classification of the irreducible $C$-representations $V$ of $G(k)$.

Theorem 3.7. The isomorphism class of $V$ is characterized by $\psi_{V}$ and $\Delta_{V} \subset \Delta_{\psi_{V}}$. For each $C$-character $\psi$ of $T(k)$ and each subset $J \subset \Delta_{\psi}$, there exists a $C$-irreducible representation $V$ of $G(k)$ such that $\psi_{V}=\psi, \Delta_{V}=J$.

Proof. [Curtis 1970, Theorem 5.7].

Definition 3.8. $\left(\psi_{V}, \Delta_{V}\right)$ are called the standard parameters of $V$. 
Example 3.9. The irreducible representations $V$ with $\psi_{V}=1$ are classified by the subsets of $\Delta$. They are sometimes called the special representations or the generalized Steinberg representations. We denote by $\mathrm{Sp}_{P}$ the representation such that $\Delta_{V}=\Delta_{M}$ for a standard parabolic group $P=M N$. The representation $\mathrm{Sp}_{G}$ is the trivial character and $\mathrm{Sp}_{B}$ is the classical Steinberg representation.

Let $P=M N$ be a standard parabolic $k$-subgroup of $G$. For $V$ an irreducible $C$-representation of $G(k)$ with standard parameters $\left(\psi_{V}, \Delta_{V}\right)$, the $C$-representation $V^{N(k)}$ of $M(k)$ is irreducible of standard parameters $\left(\psi_{V}, \Delta_{V} \cap \Delta_{M}\right)$ [Henniart and Vigneras 2011, 5.7(i)].

Proposition 3.10. The P-regular irreducible $C$-representations $V$ of $G(k)$ are in bijection with the irreducible representations of $M(k)$ by the map $V \mapsto V^{N(k)}$. Those representations $V$ with $M_{V}=M$ correspond to the characters of $M(k)$.

Proof. Fix an irreducible representation $W$ of $M(k)$ with standard parameters $\left(\psi_{W}, \Delta_{W}\right)$. For an irreducible representation $V$ of $G(k)$ with standard parameters $\left(\psi_{V}, \Delta_{V}\right)$, we have $V^{N(k)} \simeq W$ if and only if $\psi_{V}=\psi_{W}$ and $\Delta_{W}=\Delta_{V} \cap \Delta_{M}$. Moreover, $V$ is $P$-regular if and only if $\Delta_{V} \subset \Delta_{M}$. This implies the first claim, and the second one follows from Corollary 3.5.

If instead of choosing $B$, we choose the Borel subgroup $\bar{B}$ opposite to $B$, then $V$ has other parameters that we call antistandard and write $\left(\bar{\psi}_{V}, \bar{\Delta}_{V}\right)$.

Lemma 3.11. The antistandard parameters of $V$ are $\bar{\psi}_{V}=w_{0}\left(\psi_{V}\right), \bar{\Delta}_{V}=w_{0}\left(\Delta_{V}\right)$. Proof. As $\bar{B}=w_{0} B w_{0}^{-1}$, the torus $T(k)$ acts by the character $w_{0}\left(\psi_{V}\right)$ on the line $V^{\bar{U}(k)}$ and $\bar{P}_{V}=w_{0} P_{V} w_{0}^{-1}$ is the stabilizer of the line $V^{\bar{U}(k)}$. Hence, the subset $\bar{\Delta}_{V}$ of simple roots is equal to $w_{0}\left(\Delta_{V}\right) \subset-\Delta$.

The contragredient representation $V^{*}$ of $V$ is irreducible and its standard parameters are:

Lemma 3.12. $\psi_{V^{*}}=w_{0}\left(\psi_{V}\right)^{-1}, \Delta_{V^{*}}=-w_{0}\left(\Delta_{V}\right)$.

Proof. By Lemma 3.11, it is equivalent to describe the antistandard parameters $\left(\bar{\psi}_{V^{*}}, \bar{\Delta}_{V^{*}}\right)$ of $V^{*}$. The direct decomposition $V=V^{U(k)} \oplus(1-\bar{U}(k)) V$ (see Proposition 3.14 below) gives a $T(k)$-equivariant isomorphism:

$$
\left(V^{*}\right)^{\bar{U}(k)}=\left(V_{\bar{U}(k)}\right)^{*} \simeq\left(V^{U(k)}\right)^{*} .
$$

The group $T(k)$ acts on the line $V^{U(k)}$ by the character $\psi_{V}$ and on $\left(V^{U(k)}\right)^{*}$ by the character $\psi_{V}^{-1}$. Hence $\bar{\psi}_{V^{*}}=\psi_{V}^{-1}$.

The space $\left(V^{*}\right)^{\bar{U}(k)}$ is the subspace of elements on $V^{*}$ vanishing on $(1-\bar{U}(k)) V$. This space is stable by $M_{V}(k)$ because the direct decomposition of $V$ for $B$ is the same as for $P_{V}$ (Remark 3.15 below). Hence $M_{V} \bar{U} \subset \bar{P}_{V^{*}}$, or equivalently, 
$-\Delta_{V} \subset \bar{\Delta}_{V^{*}}=w_{0}\left(\Delta_{V^{*}}\right)$. As $V$ is isomorphic to the contragredient of $V^{*}$ and $-w_{0}$ is an involution on $\Delta$, we have also the inclusion in the other direction.

Remark 3.13. In general, $-w_{0}$ does not act trivially on $\Delta$ (for example for $G=$ GL(3)), and hence the stabilizer $\bar{P}_{V}$ of $V^{\bar{U}(k)}$ in $G(k)$ is not the opposite of $P_{V}$, and the $P$-regularity of $V$ is not equivalent to the $\bar{P}$-regularity of $V$. The $P$-regularity of $V$ is equivalent to the $\bar{P}$-regularity of $V^{*}$.

For a subgroup $H \subset G(k)$ and a subspace $W \subset V$, the notation $(1-H) W$ denotes the subspace of $V$ linearly generated by the elements $v-h v$ for all $h \in H$ and $v \in W$.

Proposition 3.14. We have the $M(k)$-equivariant direct decomposition

$$
V=V^{N(k)} \oplus(1-\bar{N}(k)) V^{N(k)}=V^{N(k)} \oplus(1-\bar{N}(k)) V,
$$

which gives an $M(k)$-isomorphism $V^{N(k)} \rightarrow V_{\bar{N}(k)}$.

Proof. [Cabanes and Enguehard 2004, Theorem 6.12].

Remark 3.15. The decompositions of $V$ for $P=P_{V}$ and for $P=B$ are the same, because $V^{U(k)}=V^{N_{V}(k)}$ by the definition of $P_{V}$.

Proposition 3.16. For $g \in G(k)$, the image of $g V^{U(k)}$ in $V_{\bar{N}(k)}$ is not 0 if and only if $g \in \bar{P}(k) P_{V}(k)$.

Proof. It is clear that the nonvanishing condition on $g$ depends only on $\bar{P}(k) g P_{V}(k)$ and that the image is not 0 when $g=1$ as $V^{U(k)} \subset V^{N(k)} \simeq V_{\bar{N}(k)}$ (Proposition 3.14). We prove that the image of $g V^{U(k)}$ in $V_{\bar{N}(k)}$ is 0 when $g$ does not belong to $\bar{P}(k) P_{V}(k)$. For convenience, we write in this proof $P_{V}=P^{\prime}=M^{\prime} N^{\prime}$.

a) We reduce to the case where $G_{\text {der }}$ is simply connected by choosing a $z$-extension defined over $k$,

$$
1 \rightarrow R \rightarrow G_{1} \rightarrow G \rightarrow 1,
$$

where $R \subset G_{1}$ is a central induced $k$-subtorus and $G_{1}$ is a connected reductive $k$-group with $G_{1, \text { der }}$ simply connected. The sequence of rational points

$$
1 \rightarrow R(k) \rightarrow G_{1}(k) \rightarrow G(k) \rightarrow 1
$$

is exact. The parabolic subgroups of $G_{1}$ inflated from $P, P^{\prime}$ are $P_{1}=M_{1} N$, $P_{1}^{\prime}=M_{1}^{\prime} N^{\prime}$, where $1 \rightarrow R \rightarrow M_{1} \rightarrow M \rightarrow 1$ and $1 \rightarrow R \rightarrow M_{1}^{\prime} \rightarrow M^{\prime} \rightarrow 1$ are $z$-extensions defined over $k$. We consider $V$ as an irreducible representation of $G_{1}(k)$ where $R(k)$ acts trivially. The image of $G_{1}(k)-\bar{P}_{1}(k) P_{1}^{\prime}(k)$ in $G(k)$ is $G(k)-\bar{P}(k) P^{\prime}(k)$. For $g_{1} \in G_{1}(k)-\bar{P}_{1}(k) P_{1}^{\prime}(k)$ of image $g \in G(k)-\bar{P}(k) P^{\prime}(k)$, the image of $g_{1} V^{N^{\prime}(k)}$ in $V_{\bar{N}(k)}$ is 0 if and only if the image of $g V^{N^{\prime}(k)}$ in $V_{\bar{N}(k)}$ is 0 . b) The proposition can be reformulated in terms of Weyl groups because the equality depends only on the image of $g$ in $\bar{P}(k) \backslash G(k) / P^{\prime}(k)=W_{M} \backslash W / W_{M^{\prime}}$. We denote 
by $\dot{w}$ a representative of $w \in W$ in $G(k)$. The proposition says that the image of $\dot{w} V^{N^{\prime}(k)}$ in $V_{\bar{N}(k)}$ is 0 if $w \in W$ does not belong to $W_{M} W_{M^{\prime}}$.

c) Given a), we now suppose that $G_{\text {der }}$ is simply connected. In this case, $V$ is the restriction of an irreducible algebraic representation $F(v)$ of $G$ with highest weight $v$ equal to a $q$-restricted character of $T$ [Herzig 2009, Appendix 1.3]. The stabilizer $W_{v}$ of $v$ in $W$ is $W_{M^{\prime}}$, the irreducible algebraic representation $F(v)$ of $M$ with highest weight $v$ is $F(v)^{N}$, and $F(v)^{N}$ is equal to the sum of all weight spaces $F(v)_{\mu}$ with $v-\mu \in \mathbb{Z} \Phi_{M}$; for $w \in W, w v$ is a weight of $F(v)^{N}$ if and only if $w \in W_{M} W_{M^{\prime}}$ [Herzig 2011, Lemma 2.3, and proof of Lemma 2.17 in the split case]. The quotient map $t: F(v) \rightarrow F(v)_{\bar{N}}$ restricts to an $M$-equivariant isomorphism $F(v)^{N} \rightarrow F(v)_{\bar{N}}$. We deduce that the weights of $F(v)_{\bar{N}}$ are the weights of $F(v)^{N}$ and are disjoint from the weights of the kernel of the quotient map $t$. In particular, for $w \in W$, the space $w\left(F(v)^{U}\right)$ is not in the kernel of $t$ if and only if $w \in W_{M} W_{M^{\prime}}$.

The space $V^{N(k)}$ is the restriction to $M(k)$ of $F(v)^{N}$ and the space $V_{\bar{N}(k)}$ is the restriction to $M(k)$ of $F(v)_{\bar{N}}$. This implies the proposition under the form given in b).

Corollary 3.17. Let $P^{\prime}=M^{\prime} N^{\prime}$ be another standard parabolic subgroup. The image of $g V^{N^{\prime}(k)}$ in $V_{\bar{N}(k)}$ is not 0 if and only if $g \in \bar{P}(k) P_{V}(k) P^{\prime}(k)$.

Proof. We have $V^{N^{\prime}(k)}=\sum_{h \in M^{\prime}(k)} h V^{U(k)}$ because the right-hand side is $N^{\prime}(k)$ stable and $V^{N^{\prime}(k)}$ is an irreducible representation of $M^{\prime}(k)$.

Remark 3.18. The equality $\bar{P} P_{V} P^{\prime}=\bar{P} P^{\prime}$ is equivalent to $P_{V} \subset \bar{P} P^{\prime}$. The latter inclusion is obviously true when $V$ is $P$-regular or $P^{\prime}$-regular.

In our study of Hecke operators, we will use the following particular case:

Corollary 3.19. For $g \in G(k)$, the image of $g V^{\bar{N}(k)}$ in $V_{N(k)}$ is not 0 if and only if $g \in P(k) \bar{P}_{V}(k) \bar{P}(k)$.

\section{Representations of $G(F)$}

4A. Notation. Let $C$ be an algebraically closed field of positive characteristic $p$, let $F$ be a local nonarchimedean field of finite residue field $k$ of characteristic $p$ and of cardinality $q$, of ring of integers $o_{F}$ and uniformizer $p_{F}$, and let $G$ be a reductive connected group over $F$. We fix a minimal parabolic $F$-subgroup $B$ of $G$ with unipotent radical $U$ and maximal $F$-split $F$-subtorus $S$. The group $B$ has the Levi decomposition $B=Z U$, where $Z$ is the $G$-centralizer of $S$. Let $\Phi(S, U)$ be the set of roots of $S$ in $U$ (called positive for $U$ ) and let $\Delta \subset \Phi(S, U)$ be the subset of simple roots. A parabolic $k$-subgroup $P$ of $G$ containing $B$ is called standard (for $U$ ), and has a unique Levi decomposition $P=M N$ with Levi subgroup $M$ containing $Z$ (called standard), and unipotent radical $N$. The group $(M \cap B)=Z(M \cap U)$ 
is a minimal parabolic $F$-subgroup of $M$, and $\Delta_{M}=\Delta \cap \Phi(S, M \cap U)$ is the set of simple roots of $\Phi(S, M \cap U)$. This procedure determines bijections between the subsets of $\Delta$, the standard parabolic $k$-subgroups of $G$, and their standard Levi subgroups.

The natural homomorphism $v: S(F) \rightarrow \operatorname{Hom}\left(X^{*}(S), \mathbb{Z}\right)$, where $X^{*}(S)$ is the group of $F$-characters of $S$, extends uniquely to a homomorphism $v: Z(F) \rightarrow$ $\operatorname{Hom}\left(X^{*}(S), \mathbb{Q}\right)$; its kernel is the maximal compact subgroup of $Z(F)$. For a standard Levi subgroup $M$, we denote by $Z(F)^{+N}$ the monoid of elements $z$ in $Z(F)$ which are $N$-positive, that is,

$$
a\left(v_{Z}(z)\right) \geq 0 \text { for all } a \in \Delta-\Delta_{M} .
$$

When these inequalities are strict, $z$ is called strictly $N$-positive. We denote by $Z(F)^{+\bar{N}}$ the monoid of elements in $Z(F)$ which are $\bar{N}$-positive, that is, $N$-negative,

$$
a\left(v_{Z}(z)\right) \leq 0 \quad \text { for all } a \in \Delta-\Delta_{M} .
$$

When $N=U$, we write $Z(F)^{+}:=Z(F)^{+U}$ and $Z(F)^{-}:=Z(F)^{+\bar{U}}$, and if the inequalities are strict, $z$ is called strictly positive or strictly negative. These notations extend to $M$; we write $Z(F)^{+M}=Z(F)^{+(U \cap M)}$.

In the building of the adjoint group $G_{\text {ad }}$ over $F$, we choose a special vertex in the apartment attached to $S$ and we write $K$ for the corresponding special parahoric subgroup, as in [Henniart and Vigneras 2011, 6.1]. The quotient of $K$ by its pro- $p$-radical $K_{+}$is the group of $k$-points of a connected reductive $k$-group $G_{k}$. The group $K / K_{+}$is $G_{k}(k)$. For $H=B, S, U, Z, P, M, N$, the image in $G_{k}(k)$ of $H(F) \cap K$ is the group of $k$-points of a connected $k$-group $H_{k}$. Note that $B_{k}$ is a minimal parabolic subgroup of $G_{k}, S_{k}$ is a maximal $k$-split torus in $B_{k}, Z_{k}$ (being the centralizer of $S_{k}$ in $G_{k}$ ) is a maximal $k$-subtorus of $B_{k}$, and $B_{k}=Z_{k} U_{k}$ is a Levi decomposition; moreover, there is a bijection between $\Delta$ and the set $\Delta_{k}$ of simple roots of $S_{k}$ (with respect to $U_{k}$ ), $P_{k}$ is a standard parabolic subgroup of $G_{k}$ of standard Levi subgroup $M_{k}$ and unipotent radical $N_{k}$, and the set $\Delta_{k, M_{k}}$ of simple roots of $S_{k}$ in $M_{k}$ is the image of $\Delta_{M}$ by the bijection above. We shall usually suppress the indices $k$ from the notation, write $H_{0}=H(F) \cap K$, and identify a character of $Z(k)$ (with the notations in the chapter on representations of $G(k)$ we have $T(k)=Z(k))$ with a smooth character of $Z_{0}$.

We now fix an irreducible $C$-representation $V$ of $G(k)$ with parameters $\left(\psi_{V}, \Delta_{V}\right)$ (Definition 3.8), a proper standard parabolic subgroup $P=M N$ of $G$, and an element $s \in S(F)$ central in $M(F)$ and strictly $N$-positive (and hence $U$-positive).

4B. $S^{\prime}$ is a localization. We also see $V$ as a smooth $C$-representation of $K$, trivial on $K_{+}$. We apply the generalities of the Satake homomorphisms to the group $G(F)$, the compact subgroup $K$, and the closed subgroup $P(F)=M(F) N(F)$. As $K$ is a 
special parahoric subgroup, the Iwasawa decomposition $G(F)=P(F) K$ is valid. We have a $G(F)$-equivariant linear map (Definition 2.1)

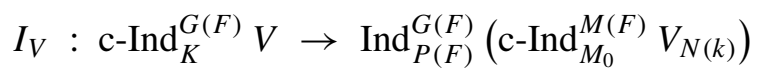

and an algebra homomorphism (Proposition 2.2)

$$
\mathscr{S}^{\prime}=\mathscr{Y}_{M, G}^{\prime}: \mathscr{H}(G(F), K, V) \rightarrow \mathscr{H}\left(M(F), M_{0}, V_{N(k)}\right),
$$

related by $I_{V}(b f)=\mathscr{S}^{\prime}(b) I_{V}(f)$ for $b \in \mathscr{H}(G(F), K, V)$ and $f$ in c- $\operatorname{Ind}_{K}^{G(F)} V$.

Proposition 4.1. The intertwiner $I_{V}$ and the algebra homomorphism $\mathscr{Y}^{\prime}$ are injective.

Proof. Apply Proposition 2.4 and [Henniart and Vigneras 2011, 7.9], giving the injectivity of the Satake homomorphism $\mathscr{S}$ appearing in Proposition 2.3 when $V, V^{\prime}$ are irreducible smooth representations of $K$ over a field of characteristic $p$.

We write $\mathscr{Y}_{G}^{\prime}=\mathscr{Y}_{Z, G}^{\prime}$ and denote by $\mathscr{S}_{G}$ the corresponding Satake homomorphisms appearing in Proposition 2.3 when $M=Z$. We analogously define $\mathscr{S}_{M}^{\prime}$ and $\mathscr{Y}_{M}$ with a commutative diagram of algebra homomorphisms:

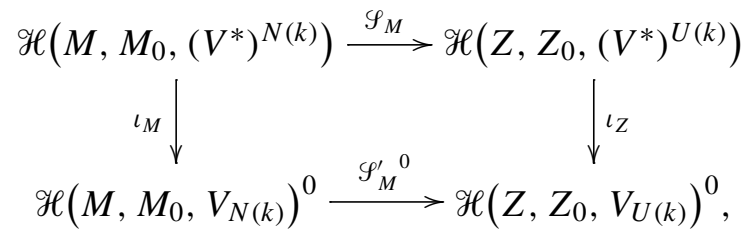

where $\left(\iota_{*}(\Phi)\right)(g)=\Phi\left(g^{-1}\right)^{t}$ for $*=M$ or $Z$ (definition before Proposition 2.3). In this diagram, $A^{0}$ denotes the opposite of an algebra $A$ and $f^{0}: A^{0} \rightarrow B^{0}$ is the algebra homomorphism $a \mapsto f^{0}(a)=f(a)$ associated to an algebra homomorphism $f: A \rightarrow B$. By the transitivity relation of the Satake homomorphisms [Henniart and Vigneras 2011, Proposition 2.8] and by Proposition 2.3, we have

$$
\mathscr{Y}_{G}^{\prime}=\mathscr{Y}_{M}^{\prime} \circ \mathscr{Y}^{\prime} .
$$

Recalling the standard parameters $\left(\psi_{V^{*}}, \Delta_{V^{*}}\right)$ of $V^{*}$, we identify $\psi_{V^{*}}$ with a smooth character of $Z_{0}$, and we denote by

$$
Z_{V^{*}}=\left\{z \in Z(F) \mid \psi_{V^{*}}\left(z x z^{-1}\right)=\psi_{V^{*}}(x) \text { for all } x \in Z_{0}\right\}
$$

the stabilizer of $\psi_{V^{*}}$ in $Z(F)$. As $\psi_{V^{*}}=w_{0}\left(\psi_{V}\right)^{-1}$ (Lemma 3.12), we have $Z_{V^{*}}=w_{0}\left(Z_{V}\right)$.

Proposition 4.2. The image of the map

$$
\mathscr{Y}_{G}^{\prime}: \mathscr{H}(G(F), K, V) \rightarrow \mathscr{H}\left(Z(F), Z_{0}, V_{U(k)}\right)
$$

is equal to $\mathscr{H}\left(Z(F)^{+} \cap Z_{V^{*}}, Z_{0}, V_{U(k)}\right)$. 
Proof. The support of a Hecke operator in $\mathscr{H}\left(Z(F), Z_{0},\left(V^{*}\right)^{U(k)}\right)$ is contained in $Z_{V^{*}}$. By [Henniart and Vigneras 2011, Theorem 1.8], the image of $\mathscr{Y}_{G}$ consists of the Hecke operators with negative support, that is, with support contained in $Z(F)^{-} \cap Z_{V^{*}}$. The image of $\iota_{Z} \circ \mathscr{S}_{G}$ consists of the Hecke operators with positive support, that is, of support in $Z(F)^{+} \cap Z_{V^{*}}$, because the inverse map permutes the monoids $Z(F)^{+}$and $Z(F)^{-}$and respects $Z_{V^{*}}$.

Analogously, the image of $\mathscr{G}_{M}^{\prime}$ is $\mathscr{H}\left(Z(F)^{+_{M}} \cap Z_{V^{*}}, Z_{0}, V_{U(k)}\right)$.

Definition 4.3. A ring morphism $f: A \rightarrow B$ is a localization at $b \in B$ if $f$ is injective, $b \in f(A)$ is central and invertible in $B$, and $B=\cup_{n \in \mathbb{N}} f(A) b^{-n}$.

There exists a unique Hecke operator $T_{Z}$ central in $\mathscr{H}\left(Z(F)^{+} \cap Z_{V^{*}}, Z_{0}, V_{U(k)}\right)$ with support $Z_{0} s$ such that $T_{Z}(s)=1$, because $s$ is $U$-positive and belongs to $S(F)$ contained in $Z_{V^{*}}$.

The algebra $\mathscr{H}\left(Z(F)^{+M} \cap Z_{V^{*}}, Z_{0}, V_{U(k)}\right)$ is the localization of

$$
\mathscr{H}\left(Z(F)^{+} \cap Z_{V^{*}}, Z_{0}, V_{U(k)}\right)
$$

at $T_{Z}$ because, for any $U \cap M$-positive element $z \in Z(F)$, there exists a positive integer $n$ such that $s^{n} z$ belongs to $Z(F)^{+}$, because $s \in S(F)$ is strictly $N$-positive.

Definition 4.4. As $s$ is central in $M(F)$ and contained in $Z_{V^{*}}$, there exists a unique Hecke operator $T_{M}$ in $\mathscr{H}\left(M(F), M_{0}, V_{N(k)}\right)$ with support $M_{0} s$ with value $\operatorname{id}_{V_{N(k)}}$ at $s$.

The Hecke operator $T_{M}$ is central and invertible in $\mathscr{H}\left(M(F), M_{0}, V_{N(k)}\right)$; it acts on c-Ind $M_{M_{0}}^{M(F)} V_{\bar{N}(k)}$ by $T_{M}\left([1, \bar{v}]_{M_{0}}\right)=s^{-1}[1, \bar{v}]_{M_{0}}$ for $v \in V$. We also denote by $T_{M}$ the $G(F)$-endomorphism of

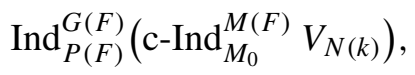

such that $T_{M}(f)(g)=T_{M}(f(g))$ for $f \in \operatorname{Ind}_{P(F)}^{G(F)}\left(\mathrm{c}-\operatorname{Ind}_{M_{0}}^{M(F)} V_{N(k)}\right)$ and $g \in G(F)$. Using Proposition 2.2, we see that

$$
\mathscr{\varphi}_{M}^{\prime}\left(T_{M}\right)=T_{Z},
$$

because $(U \cap M)(F) z \cap M_{0} s=\left((U \cap M)(F) z s^{-1} \cap M_{0}\right) s=\left(U_{0} \cap M_{0}\right) z$ if $z s^{-1} \in Z_{0}$ and is 0 otherwise. The Hecke operator $T_{M}$ belongs to the image of $\mathscr{Y}^{\prime}$, because $T_{Z}$ belongs to the image of $\mathscr{Y}_{G}^{\prime}$ by construction, $\mathscr{S}^{\prime}$ is injective, and we have (10), (9). We have shown:

Proposition 4.5. The map $\mathscr{S}^{\prime}$ is a localization at $T_{M}$.

In (7), we consider the map $I_{V}$ as a $C[T]$-linear map, $T$ acting on the left side by $\left(\mathscr{S}^{\prime}\right)^{-1}\left(T_{M}\right)$ and on the right side by $T_{M}$. By Proposition 4.5 , the localization of 
$I_{V}$ at $T$ is the $\left(G(F), \mathcal{H}\left(M(F), M_{0}, V_{N(k)}\right)\right)$-equivariant map

$$
\begin{aligned}
& \Theta: \mathscr{H}\left(M(F), M_{0}, V_{N(k)}\right) \otimes_{\mathscr{H}(G(F), K, V), \mathscr{S}^{\prime}} \mathrm{c}-\operatorname{Ind}_{K}^{G(F)} V \\
& \rightarrow \operatorname{Ind}_{P(F)}^{G(F)}\left(\operatorname{c}^{-\operatorname{Ind}_{M}} M_{M_{0}}^{M(F)} V_{N(k)}\right) .
\end{aligned}
$$

The map $\Theta$ is injective because $I_{V}$ is injective (Proposition 4.1). Our main theorem is:

Theorem 4.6. $\Theta$ is surjective if $V$ is $\bar{P}$-regular.

The theorem will follow from Corollary 6.5 and Proposition 5.4.

4C. Decomposition of the intertwiner. Following Herzig, we write the intertwiner $I_{V}$ as a composite of two $G(F)$-equivariant linear maps

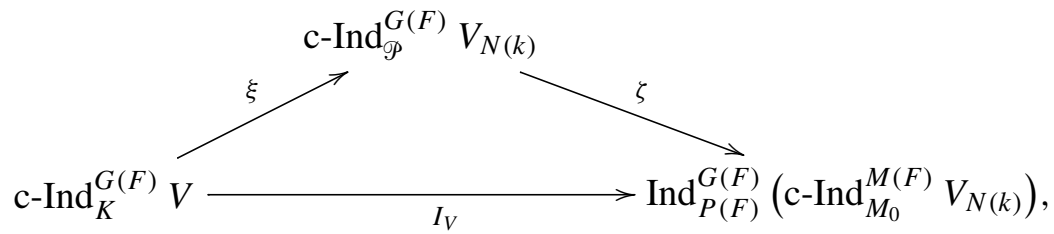

which we now define. In this diagram, $\mathscr{P}$ is the inverse image in $K$ of $P(k)$. The image of $\mathscr{P}$ in $G(k)$ is $P(k)$ by [Bruhat and Tits 1984, 5.1.22]; $\mathscr{P}$ is a parahoric subgroup of $G(F)$.

Lemma 4.7. The parahoric subgroup $\mathscr{P}$ admits an Iwahori decomposition with respect to $M$,

$$
\mathscr{P}=N_{0} M_{0} \bar{N}_{0+}, \quad \bar{N}_{0,+}:=\bar{N}(F) \cap K_{+},
$$

with any order of the factors.

Proof. This decomposition is well known, but at the referee's suggestion, we outline a proof. By [Bruhat and Tits 1984, 4.6.4 and 5.1.31], $K_{+}=U_{0+} Z_{0+} \bar{U}_{0+}$, with the sign + indicating the intersection with $K_{+}$as above. As $M_{0}$ is the parahoric subgroup of $M(F)$ fixing our special point, we have $M_{0+}=\left(U_{0+} \cap M_{0}\right) Z_{0+}\left(\bar{U}_{0+} \cap\right.$ $M_{0}$ ). It follows that $K_{+}=N_{0+} M_{0+} \bar{N}_{0+}$. From [Henniart and Vigneras 2011, Theorem 6.5], we have $\mathscr{P}=N_{0} M_{0} K_{+}$, and so $\mathscr{P}=N_{0} M_{0} N_{0+} M_{0+} \bar{N}_{0+}$. As $M_{0}$ normalizes $N_{0}, \bar{N}_{0}$ and $K_{+}$, it normalizes also $N_{0+}$ and $\bar{N}_{0+}$, and we have the decomposition $\mathscr{P}=N_{0} M_{0} \bar{N}_{0+}$ with any order of the factors.

The transitivity of compact induction implies that

$$
\mathrm{c}-\operatorname{Ind}_{\mathscr{P}}^{G(F)} V_{N(k)} \simeq \mathrm{c}-\operatorname{Ind}_{K}^{G(F)}\left(\mathrm{c}-\operatorname{Ind}_{P(k)}^{G(k)} V_{N(k)}\right) .
$$


Definition 4.8. The map $\xi$ is the image by the compact induction functor c-Ind $K_{K}^{G}$ of the natural embedding $V \rightarrow \mathrm{c}-\operatorname{Ind}_{P(k)}^{G(k)} V_{N(k)}$.

For $v \in V, \xi\left([1, v]_{K}\right)$ is the function in c- $\operatorname{Ind}_{\mathscr{F}}^{G(F)} V_{N(k)}$ with support contained in $K$ and value $\overline{k v}$ at $k \in K$.

Proposition 4.9. There is a unique $G(F)$-equivariant map

$$
\zeta: \mathrm{c}-\operatorname{Ind}_{\mathscr{P}}^{G(F)} V_{N(k)} \rightarrow \operatorname{Ind}_{P(F)}^{G(F)}\left(\mathrm{c}-\operatorname{Ind}_{M_{0}}^{M(F)} V_{N(k)}\right),
$$

which for $v \in V$, sends $[1, \bar{v}]_{\mathscr{P}}$ to the function $f_{\bar{v}}$ with support contained in

$$
P(F) \mathscr{P}=P(F) \bar{N}_{0,+}
$$

and constant value $[1, \bar{v}]_{M_{0}}$ on $\bar{N}_{0,+}$.

Proof. The uniqueness is clear because the functions $[1, \bar{v}]_{\mathscr{P}}$ for $v \in V$ generate the representation c-Ind $\operatorname{IP}^{G(F)} V_{N(k)}$. The existence can be proved directly, but we can also apply the considerations of the beginning of Section 2 with $V^{\prime}:=\mathrm{c}-\operatorname{Ind}_{\mathscr{P}}^{K}\left(V_{N(k)}\right)$ instead of $V$ and $W=\mathrm{c}-\operatorname{Ind}_{M_{0}}^{M(F)} V_{N(k)}$.

The value at 1 from $V^{\prime}$ to $V_{N(k)}$ factorizes through the quotient map $v^{\prime} \mapsto \overline{v^{\prime}}$ from $V^{\prime}$ to $V_{N(k)}^{\prime}$ and defines an $M_{0}$-equivariant map $r: V_{N(k)}^{\prime} \rightarrow V_{N(k)}$, such that $r\left(\overline{v^{\prime}}\right)=v^{\prime}(1)$ for all $v^{\prime} \in V^{\prime}$. The image of $r$ by the compact induction functor from $M_{0}$ to $M(F)$ is an element in

$$
\operatorname{Hom}_{M(F)}\left(\mathrm{c}-\operatorname{Ind}_{M_{0}}^{M(F)} V_{N(k)}^{\prime}, \mathrm{c}-\operatorname{Ind}_{M_{0}}^{M(F)} V_{N(k)}\right)
$$

which corresponds by the isomorphism (2) to an element in

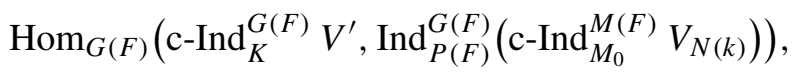

sending $\left[1, v^{\prime}\right]_{K}$ to the unique function $\varphi_{v^{\prime}}$ with value on $k \in K$ equal to

$$
\left.\left[1, r\left(\overline{k v^{\prime}}\right)\right]_{M_{0}}=\left[1, v^{\prime}(k)\right)\right]_{M_{0}},
$$

for all $v^{\prime} \in V^{\prime}$. Applying the transitivity of the compact induction functor to c-Ind ${ }_{K}^{G(F)} V^{\prime}$, we obtain the element

$$
\zeta \in \operatorname{Hom}_{G(F)}\left(\mathrm{c}-\operatorname{Ind}_{\mathscr{P}}^{G(F)} V_{N(k)}, \operatorname{Ind}_{P(F)}^{G(F)}\left(\mathrm{c}-\operatorname{Ind}_{M_{0}}^{M(F)} V_{N(k)}\right)\right)
$$

of the proposition. For $v \in V$ with image $\bar{v}$ in $N_{N(k)}$, the morphism $\zeta$ sends $[1, \bar{v}]_{\mathscr{P}}$ to $\varphi_{v^{\prime}}$, where $v^{\prime} \in V^{\prime}$ is the function on $K$ of support $\mathscr{P}$ and equal to $\bar{v}$ at 1 . It remains to check that $\varphi_{v^{\prime}}$ is equal to the function $f_{\bar{v}}$ given in the proposition. Indeed, the support of the function $\varphi_{v^{\prime}} \in \mathrm{c}-\operatorname{Ind}_{\mathscr{P}}^{G(F)} V_{N(k)}$ is contained in $P(F) \mathscr{P}$, we have $P(F) \mathscr{P}=P(F) \bar{N}_{0,+}$ by the Iwahori decomposition of $\mathscr{P}$, and for $k \in \bar{N}_{0,+}$ we have $v^{\prime}(k)=\bar{v}$. 
Remark 4.10. Later we will use that, for $g \in G(F), \zeta\left(g^{-1}[1, \bar{v}]_{\odot}\right)$ has support in $P(F) \mathscr{P} g$ which contains 1 if and only if $g \in \mathscr{P} P(F)$. Thus, for $f \in \mathrm{c}-\operatorname{Ind}_{\mathscr{P}}^{G(F)} V_{N(k)}$, the element $\zeta(f)(1)$ depends only on the restriction of $f$ to $\mathscr{P} P(F)$.

Lemma 4.11. $I_{V}=\zeta \circ \xi$.

Proof. This is clear from the definitions of $I_{V}, \xi, \zeta$.

Remark 4.12. The map $\xi$ is injective because $I_{V}$ is injective (Proposition 4.1). We can give a direct proof: As $V$ is irreducible and $V_{N(k)} \neq 0$, the map $V \rightarrow$ c-Ind ${ }_{P(k)}^{G(k)} V_{N(k)}$ is injective. As the functor c-Ind ${ }_{K}^{G}$ is exact, the map $\xi$ is injective.

The map $\xi$ is not surjective because the map $V \rightarrow \mathrm{c}-\operatorname{Ind}_{P(k)}^{G(k)} V_{N(k)}$ is not surjective, as $P \neq G$ by our running hypothesis. This can be seen by taking fixed points under $U(k)$.

\section{Hecke operators}

In this chapter, we introduce some Hecke operators associated to our fixed element $s \in S(F)$ central in $M(F)$ and strictly $N$-positive, and we show the compatibility of these Hecke operators with the maps $\xi, \zeta, \mathscr{Y}^{\prime}$ (sometimes we need to suppose that $V$ is $\bar{P}$-regular).

The space of $G(F)$-equivariant homomorphisms from c- $\operatorname{Ind}_{K}^{G(F)} V$ to

$$
\mathrm{c}-\operatorname{Ind}_{\mathscr{P}}^{G(F)} V_{N(k)},
$$

is isomorphic to the space $\mathscr{H}\left(G(F), K, \mathscr{P}, V, V_{N(k)}\right)$ of functions $\Phi: G(F) \rightarrow$ $\operatorname{Hom}_{C}\left(V, V_{N(k)}\right)$ satisfying

(i) $\Phi\left(j g j^{\prime}\right)=j \circ \Phi(g) \circ j^{\prime}$ for $j \in \mathscr{P}, j^{\prime} \in K$,

(ii) $\Phi$ vanishes outside finitely many double cosets $\mathscr{P} g K$.

We call $\Phi$ a Hecke operator. We shall usually use the same notation for the Hecke operator and for the corresponding $G(F)$-equivariant homomorphism, defined by: for all $v \in V$,

$$
[1, v]_{K} \rightarrow \sum_{g \in \mathscr{P} \backslash G(F)} g^{-1}[1, \Phi(g)(v)]_{\mathscr{P}}
$$

The map $\xi$ corresponds to the Hecke operator with support $K$ and value at 1 the projection $V \rightarrow V_{N(k)}$ given by $v \mapsto \bar{v}$.

In the same way, the space of $G(F)$-equivariant homomorphisms

$$
\mathrm{c}-\operatorname{Ind}_{\mathscr{P}}^{G(F)} V_{N(k)} \rightarrow \mathrm{c}-\operatorname{Ind}_{K}^{G(F)} V
$$

corresponds to a space $\mathscr{H}\left(G(F), \mathscr{P}, K, V_{N(k)}, V\right)$ of functions from $G(F)$ to

$$
\operatorname{Hom}_{C}\left(V_{N(k)}, V\right) \text {. }
$$


5A. Definition of Hecke operators. Recall (Proposition 3.14) that the quotient map $v \mapsto \bar{v}$ from $V$ to $V_{N(k)}$ induces an isomorphism $V^{\bar{N}(k)} \rightarrow V_{N(k)}$. We write $\varphi: V_{N(k)} \rightarrow V^{\bar{N}(k)}$ for the reciprocal isomorphism. Since $s \in S(F)$ is $U$-positive and belongs to $Z_{V^{*}}$, we deduce from [Henniart and Vigneras 2011, 7.3 Lemma 1]:

Proposition 5.1. There exists a unique Hecke operator $T_{G}$ in $\mathscr{H}(G(F), K, V)$ with support $K s K$ such that $T_{G}(s) \in \operatorname{End}_{C}(V)$ sends $v \in V$ to $\varphi(\bar{v})$.

The Hecke operator $T_{M}$ (Definition 4.4) could have been defined in the same way. We shall prove later that $\mathscr{Y}^{\prime}\left(T_{G}\right)=T_{M}$ when $V$ is $\bar{P}$-regular. We define now Hecke operators $T_{\mathscr{P}}$ and $T_{K, \mathscr{P}}$ generalizing $T_{G}$ and $T_{M}$.

Proposition 5.2. (i) There is a unique Hecke operator $T_{\mathscr{P}}$ in $\mathscr{H}\left(G(F), \mathscr{P}, V_{N(k)}\right)$ with support $\mathscr{P}_{S} \mathscr{P}$ and value at $s$ the identity of $V_{N(k)}$.

(ii) There is a unique Hecke operator $T_{K, \mathscr{P}}$ in $\mathscr{H}\left(G(F), \mathscr{P}, K, V_{N(k)}, V\right)$ with support $K s \mathscr{P}$ such that $T_{K, \mathscr{P}}(s): V_{N(k)} \rightarrow V$ sends $\bar{v}$ to $\varphi(\bar{v})$.

Proof. (i) By the condition (i) for Hecke operators, we have to check that for $h, h^{\prime} \in \mathscr{P}$, the relation $h^{\prime} s=s h$ implies that the actions of $h$ and of $h^{\prime}$ on $V_{N(k)}$ are the same. We use the Iwahori decomposition (13):

$$
\mathscr{P}=\bar{N}_{0+} M_{0} N_{0}
$$

Decomposing $h=\bar{n} m n$, we have $h^{\prime}=s \bar{n} s^{-1} m s n s^{-1}$, since $s$ is central in $M(F)$. Because $s$ is $N$-positive, $s n s^{-1} \in N_{0}$ and the condition $h^{\prime} \in \mathscr{P}$ means that $s \bar{n} s^{-1} \in$ $\bar{N}_{0+}$. Consequently, both $h$ and $h^{\prime}$ act as $m$ on $V_{N(k)}$.

(ii) We now have to check that for $h^{\prime} \in K, h \in \mathscr{P}$, the relation $h^{\prime} s=s h$ implies that $h^{\prime} \varphi(\bar{v})=\varphi(\overline{h v})$ for all $v \in V$. Writing as above $h=\bar{n} m n$, the condition $h^{\prime} \in K$ means $s \bar{n} s^{-1} \in \bar{N}(F) \cap K=\bar{N}_{0}$, so that $\bar{n}$ belongs to $\bar{N}_{0+}$ because $s$ is strictly $N$-positive. Then $\varphi(\overline{h v})=\varphi(\overline{m n v})=m \varphi(\overline{n v})=m \varphi(\bar{v})$. But $s n s^{-1}$ is in $N_{0+}$ again because $s$ is strictly $N$-positive and $h^{\prime} \varphi(\bar{v})=m \varphi(\bar{v})$ too.

Remark 5.3. We note that, for $v \in V$ :

- $T_{\mathscr{P}}\left([1, \bar{v}]_{\mathscr{P}}\right)$ is the function in c-Ind $\operatorname{In}_{\mathscr{P}}^{G(F)} V_{N(k)}$ with support $\mathscr{P}_{S} \mathscr{P}$ and value $\bar{v}$ on $s \bar{N}_{0+}$.

- $T_{K, \mathscr{P}}\left([1, \bar{v}]_{\mathscr{P}}\right)$ is the function in c-Ind ${ }_{K}^{G(F)} V$ with support $K s \mathscr{P}$ and value $\varphi(v)$ on $s \bar{N}_{0+}$.

- $T_{G}\left([1, v]_{K}\right)$ is the function in c-Ind ${ }_{K}^{G(F)} V$ with support contained in $K s K$ and value $\varphi(\overline{h v})$ on $s h$ for all $h \in K$.

5B. Compatibilities between Hecke operators. In this section, following Herzig's method, we prove: 
Proposition 5.4. (i) The diagram on the left

(16)
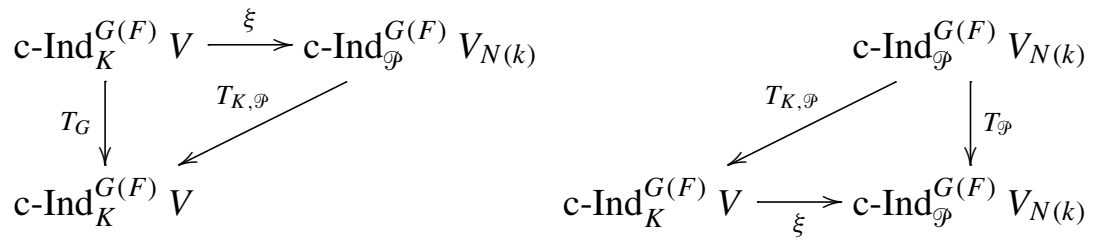

is commutative; the diagram on the right is commutative when $V$ is $\bar{P}$-regular.

(ii) The diagram

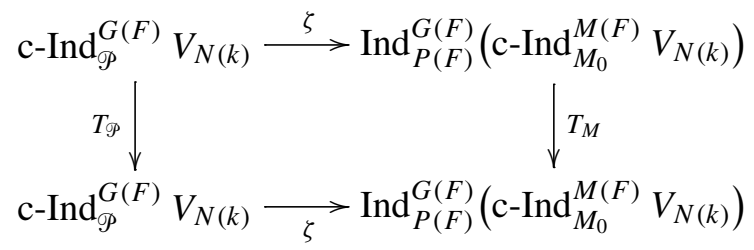

is commutative.

(iii) $\mathscr{S}^{\prime}\left(T_{G}\right)=T_{M}$ when $V$ is $\bar{P}$-regular.

By (15), the $G(F)$-homomorphisms corresponding to $\xi, T_{G}, T_{\mathscr{P}}$ and $T_{K, \mathscr{P}}$ are characterized by the following formulas, for $v \in V$ :

$$
\begin{aligned}
\xi:[1, v]_{K} & \mapsto \sum_{g \in \mathscr{P} \backslash K} g^{-1}[1, \overline{g v}]_{\mathscr{P}}, \\
T_{G}:[1, v]_{K} & \mapsto \sum_{g \in K \backslash K s K} g^{-1}\left[1, T_{G}(g)(v)\right]_{K}, \\
T_{\mathscr{P}}:[1, \bar{v}]_{\mathscr{P}} & \rightarrow \sum_{g \in \mathscr{P} \backslash \mathscr{P}_{S} \mathscr{P}} g^{-1}\left[1, T_{\mathscr{P}}(g)(\bar{v})\right]_{\mathscr{P}}, \\
T_{K, \mathscr{P}}:[1, \bar{v}]_{\mathscr{P}} & \mapsto \sum_{g \in K \backslash K S \mathscr{P}} g^{-1}\left[1, T_{K, \mathscr{P}}(g)(\bar{v})\right]_{K} .
\end{aligned}
$$

To prove the proposition, it is useful first to simplify these formulas.

Lemma 5.5. We have

$$
\begin{aligned}
T_{\mathscr{P}}:[1, \bar{v}]_{\mathscr{P}} & \mapsto \sum_{\bar{n} \in s^{-1} \bar{N}_{0+} s \backslash \bar{N}_{0+}} \bar{n}^{-1} s^{-1}[1, \bar{v}]_{\mathscr{P}}, \\
T_{K, \mathscr{P}}:[1, \bar{v}]_{\mathscr{P}} & \mapsto \sum_{\bar{n} \in s^{-1} \bar{N}_{0} s \backslash \bar{N}_{0+}} \bar{n}^{-1} s^{-1}[1, \varphi(\bar{v})]_{K}, \\
T_{G}:[1, v]_{K} & \mapsto \sum_{h \in \mathscr{P} \backslash K} h^{-1} \sum_{\bar{n} \in s^{-1} \bar{N}_{0} s \backslash \bar{N}_{0+}} \bar{n}^{-1} s^{-1}[1, \varphi(\overline{h v})]_{K} .
\end{aligned}
$$


Proof. By the Iwahori decomposition $\mathscr{P}=N_{0} M_{0} \bar{N}_{0+}$, we get that $\mathscr{P}_{S} \mathscr{P}=\mathscr{P}_{S} \bar{N}_{0+}$, because $s N_{0} s^{-1} \subset N_{0}$ and $s M_{0} s^{-1}=M_{0}$. Consequently, the map $\bar{n} \mapsto s \bar{n}$ induces a bijection of $s^{-1} \bar{N}_{0+} s \backslash \bar{N}_{0+}$ onto $\mathscr{P} \backslash \mathscr{P} S \mathscr{P}$. Since $\bar{N}_{0+}$ acts trivially on $V_{N(k)}$, we get the formula for $T_{\mathscr{P}}$.

A similar reasoning gives that $K s \mathscr{P}=K s \bar{N}_{0+}$ and that $\bar{n} \mapsto s \bar{n}$ induces a bijection of $s^{-1} \bar{N}_{0} \backslash \backslash \bar{N}_{0+}$ onto $K \backslash K s \mathscr{P}$. This implies the formula for $T_{K, \mathscr{P}}$.

To simplify the formula for $T_{G}$, we note that the map $h \mapsto s h$ induces a bijection from $\left(K \cap s^{-1} K s\right) \backslash K$ onto $K \backslash K s K$. But $K \cap s^{-1} K s$ is contained in $\mathscr{P}$ by [Henniart and Vigneras 2011, Proposition 6.13], so that we can perform the sum in $T_{G}$ as a sum over $\left(K \cap s^{-1} K s\right) \backslash \mathscr{P}$ followed by a sum over $\mathscr{P} \backslash K$. By what we said in the previous paragraph, the inclusion $\bar{N}_{0+} \subset \mathscr{P}$ induces a bijection of $s^{-1} \bar{N}_{0} s \backslash \bar{N}_{0,+}$ onto $\left(K \cap s^{-1} K s\right) \backslash \mathscr{P}$, so that we finally get the formula for $T_{G}$.

We now give the proof of Proposition 5.4.

Proof. From the formulas for $T_{G}, T_{K, \oplus}$ in Lemma 5.5 and the formula for $\xi$, we immediately get

$$
T_{G}=T_{K, \mathscr{P}} \circ \xi,
$$

so that the left diagram in Proposition 5.4(i) is indeed commutative.

The elements $[1, \bar{v}]_{\mathscr{P}}$ for $v \in V$ generate the representation $\mathrm{c}-\operatorname{Ind}_{\mathscr{P}}^{G(F)} V_{N(k)}$, and to prove the commutativity of the diagram in Proposition 5.4(ii), it thus suffices to prove for $v \in V$ the equality

$$
\left(T_{M} \circ \zeta\right)\left([1, \bar{v}]_{\mathscr{P}}\right)=\left(\zeta \circ T_{\mathscr{P}}\right)\left([1, \bar{v}]_{\mathscr{P}}\right) .
$$

From the value of $\zeta\left([1, \bar{v}]_{\mathscr{P}}\right)$ for $v \in V$ given in Proposition 4.9 and from

$$
T_{M}\left([1, \bar{v}]_{M_{0}}\right)=s^{-1}[1, \bar{v}]_{M_{0}},
$$

we see that the function $\left(T_{M} \circ \zeta\right)\left([1, \bar{v}]_{\mathscr{P}}\right)$ vanishes outside $P \bar{N}_{0+}$ and has constant value $s^{-1}[1, \bar{v}]_{M_{0}}$ on $\bar{N}_{0+}$. From the formula for $T_{\mathscr{P}}$ in Lemma 5.5, we have

$$
\left(\zeta \circ T_{\mathscr{P}}\right)\left([1, \bar{v}]_{\mathscr{P}}\right)=\sum_{\bar{n} \in s^{-1} \bar{N}_{0+} s \backslash \bar{N}_{0+}} \bar{n}^{-1} s^{-1} \zeta\left([1, \bar{v}]_{\mathscr{P}}\right),
$$

and with the value of $\zeta\left([1, \bar{v}]_{\mathscr{P}}\right)$, we see that this function is indeed the function $\left(T_{M} \circ \zeta\right)\left([1, \bar{v}]_{\mathscr{P}}\right)$ described above, so that the diagram in Proposition 5.4(ii) is commutative.

Let us turn to the proof of the commutativity of the diagram on the right in Proposition 5.4(i). We now assume that $V$ is $\bar{P}$-regular. From the formulas for $T_{K, \mathscr{P}}$ in Lemma 5.5, we have, for $v \in V$,

$$
\xi \circ T_{K, \mathscr{P}}:[1, \bar{v}]_{\mathscr{P}} \mapsto \sum_{\bar{n} \in s^{-1} \bar{N}_{0} s \backslash \bar{N}_{0+}} \bar{n}^{-1} s^{-1} \sum_{h \in \mathscr{P} \backslash K} h^{-1}[1, \overline{h \varphi(\bar{v})}]_{\mathscr{P}} .
$$


We have seen that for $h \in K$, the image of $h V^{\bar{N}(k)}$ in $V_{N(k)}$ is 0 unless $h$ belongs to $\mathscr{\mathscr { P }} \bar{P}$ (Corollary 3.19), so that the inner sum can be restricted to $h \in \bar{N}_{0,+} \backslash \bar{N}_{0}$. Now $\bar{n}^{-1} s^{-1} h^{-1}=\bar{n}^{-1} s^{-1} h^{-1} s s^{-1}$ and $s^{-1} h s$ runs through $s^{-1} \bar{N}_{0,+} s \backslash s^{-1} \bar{N}_{0} s$, which gives the result

$$
\xi \circ T_{K, \mathscr{P}}\left([1, \bar{v}]_{\mathscr{P}}\right)=T_{\mathscr{P}}\left([1, \bar{v}]_{\mathscr{P}}\right) .
$$

We finally prove $\mathscr{Y}^{\prime}\left(T_{G}\right)=T_{M}$, still assuming that $V$ is $\bar{P}$-regular. We have just proved $\xi \circ T_{K, \mathscr{P}}=T_{\mathscr{P}}$ and previously we got $T_{K, \mathscr{P}} \circ \xi=T_{G}$, so we deduce $\xi \circ T_{G}=T_{\mathscr{P}} \circ \xi$. We also proved $\zeta \circ T_{\mathscr{P}}=T_{M} \circ \zeta$, so we obtain

$$
\zeta \circ \xi \circ T_{G}=\zeta \circ T_{\mathscr{P}} \circ \xi=T_{M} \circ \zeta \circ \xi
$$

that is, $I_{V} \circ T_{G}=T_{M} \circ I_{V}$. Applying $j_{V}$ and Definition 2.1, this implies $\mathscr{Y}^{\prime}\left(T_{G}\right)=T_{M}$.

Note that the trivial representation $V$ is not $\bar{P}$-regular, as $M \neq G$ by our running hypothesis; however, we can still have $\mathscr{G}^{\prime}\left(T_{G}\right)=T_{M}$ when the representation $V$ is the trivial representation. We now present some examples of that phenomenon (the referee remarks that even more examples result from [Herzig 2011, Proposition 5.1]).

Example 5.6. Take $G=\mathrm{GL}(2,-), Z_{G}$ the center, $M$ the diagonal group, $B=N M$ the upper triangular subgroup, $K=\mathrm{GL}\left(2, o_{F}\right)$, and

$$
s_{p}:=\left(\begin{array}{cc}
p_{F} & 0 \\
0 & 1
\end{array}\right) .
$$

The monoid of strictly positive elements in $M(F)$ is $\cup_{n \geq 1} s_{p}^{n} Z_{G}(F) M_{0}$, where $M_{0}=M(F) \cap K$. An irreducible smooth $C$-representation $V$ of $K$ is $B$-regular if and only if it is $\bar{B}$-regular if and only if it is not 1-dimensional. For $g \in G(F)$, we denote by $T_{g}$ the characteristic function of $K g K$ in the Hecke $C$-algebra $\mathscr{H}(G(F), K, C) \simeq$ $C[K \backslash G(F) / K]$ of (the trivial $C$-representation of) $K$ in $G(F)$. For $t \in M(F)$, we denote by $\tau_{t}$ the characteristic function of $t M_{0}$ in the Hecke algebra

$$
\mathscr{H}\left(M(F), M_{0}, C\right) \simeq C\left[M(F) / M_{0}\right] .
$$

Claim. When $s \in M(F)$ is strictly positive, we have $\mathscr{S}^{\prime}\left(T_{s}\right)=\tau_{s}$ if and only if $s \in s_{p} Z_{G} M_{0}$.

Proof. By [Barthel and Livné 1994, Proposition 8], the characteristic function $T_{n}$ of $Z_{G}(F) K s_{p}^{n} K$ in the Hecke algebra $\mathscr{H}\left(G(F), Z_{G}(F) K, C\right)$ satisfies the relations

$$
T_{n}=T_{1}^{n}-T_{1}^{n-2} \text { for } n \geq 2 .
$$

The natural surjective $G(F)$-equivariant map

$$
\sigma: \mathrm{c}-\operatorname{Ind}_{K}^{G(F)} C \rightarrow \mathrm{c}-\operatorname{Ind}_{Z_{G}(F) K}^{G(F)} C, \quad 1_{K} \mapsto 1_{Z_{G} K},
$$


satisfies $\sigma \circ T_{s}=T_{n} \circ \sigma$ when $s \in s_{p}^{n} p_{F}^{\mathbb{Z}_{G}} M_{0}, n \geq 1$.

Indeed, we write $K s K$ as a disjoint union of cosets $K b_{i} p_{F}^{r}$, where $s \in s_{p}^{n} p_{F}^{r} M_{0}$ and $b_{i} \in B(F)$. For $f \in \mathrm{c}-\operatorname{Ind}_{K}^{G(F)} C$, we have

$$
T_{s}(f)=\sum_{i} p_{F}^{-r} b_{i}^{-1} f \quad \text { and } \quad\left(\sigma \circ T_{s}\right)(f)=\sum_{i} b_{i}^{-1} \sigma(f) .
$$

The double coset $Z_{G}(F) K s_{p}^{n} K$ is the union of the cosets $Z_{G}(F) K b_{i}$. The union remains disjoint because the equality of cosets $Z_{G}(F) K b_{i}=Z_{G}(F) K b_{j}$, equivalent to $b_{j} b_{i}^{-1} k=z$ for some $z \in Z_{G}(F), k \in K$, implies that the determinant of $z$ is a unit. When this holds, $z \in M_{0} \cap Z_{G}$ and $K b_{i}=K b_{j}$. For $\varphi \in \mathrm{c}-\operatorname{Ind}_{Z_{G}(F) K}^{G(F)} C$, we have

$$
T_{n}(\varphi)=\sum_{i} b_{i}^{-1} \varphi \quad \text { and } \quad\left(T_{n} \circ \sigma\right)(f)=\sum_{i} b_{i}^{-1} \sigma(f) .
$$

We deduce $\sigma \circ T_{s}=T_{n} \circ \sigma$. Then the relation (21) implies that $T_{S_{p}^{n}}$ is different from $T_{s_{p}}^{n}$ when $n \geq 2$.

The value of $\mathscr{S}^{\prime}\left(T_{s}\right)$ at $t \in M(F)$ is the image in $C$ of the number of $b \in F / o_{F}$ such that $n_{b} t \in K s K$, where

$$
n_{b}:=\left(\begin{array}{ll}
1 & b \\
0 & 1
\end{array}\right)
$$

The double coset $K s_{p} K$ is the disjoint union of $K s_{p}$ and of $K\left(\begin{array}{cc}1 & a \\ 0 & p_{F}\end{array}\right)$ for $a$ in a system of representatives of $o_{F} / p_{F} o_{F}$. The characteristic of $C$ being $p$, we deduce that $\mathscr{S}^{\prime}\left(T_{s_{p}}\right)=\tau_{s_{p}}$. Then we obtain $\mathscr{S}^{\prime}\left(T_{s_{p}^{n}}\right) \neq \tau_{s_{p}^{n}}$ when $n \geq 2$, because $\mathscr{S}^{\prime}$ is an injective algebra homomorphism and $T_{s_{p}^{n}} \neq T_{s_{p}}^{n}$. Our claim is proved for $s=s_{p}^{n}$ and $n \geq 1$. The general case $s=s_{p}^{n} p_{F}^{r} t_{0}$ with $r \in \mathbb{Z}, t_{0} \in M_{0}$, reduces easily to this case.

Example 5.7. Let $D$ be a quaternion division algebra over $F$. We write $O$ for the ring of integers of $D$, and $v$ for its normalized valuation; we choose a uniformizer $p_{D}$ of $D$ such that $p_{D}^{2}=p_{F}$ is a uniformizer of $F$; the residue field $k_{D}$ of 0 is a quadratic extension of the residue field $k$ of $F$. We take for $G$ the group such that $G(F)=\mathrm{GL}(2, D)$, for $S$ the group such that $S(F)$ is the group of diagonal matrices with coefficients in $F^{*}$, and for $B=M N$ the groups such that $M(F)$ is the group of diagonal matrices and $B(F)$ is the upper triangular subgroup of $\operatorname{GL}(2, D)$.

Let $K=\mathrm{GL}(2,0)$; the quotient of $K$ by its pro- $p$-radical is isomorphic to $\mathrm{GL}\left(2, k_{D}\right)$. The Cartan decomposition says that $G(F)$ is the disjoint union of the double cosets $K d_{a, b} K$, for integers $a, b \in \mathbb{Z}$ with $a \geq b$, where $d_{a, b}$ is the diagonal matrix with entries $p_{D}^{a}$ and $p_{D}^{b}$ down the diagonal. The strictly positive elements of $M(F)$ are those of the form $s=m_{0} d_{a, b}$, for $a, b \in \mathbb{Z}$ with $a>b$ and $m_{0} \in M_{0}=M(F) \cap K$. 
An irreducible $C$-representation of $\operatorname{GL}\left(2, k_{D}\right)$ which is not $\bar{B}$-regular has dimension 1 and is given by a character $g \mapsto(\epsilon \circ$ det $)(g)$, where $\epsilon: k_{D}^{*} \rightarrow C^{*}$ is a character. We identify $\epsilon$ o det with an irreducible smooth $C$-character of $K$ and $\epsilon$ with a smooth $C$-character of $0^{*}$.

The reduction of the conjugation by $p_{D}$ on $\mathbb{O}$ induces the nontrivial automorphism $\sigma$ of $k_{D} / k$. The character $\epsilon$ of $0^{*}$ extends to a character of $D^{*}$ exactly when $\epsilon$ is invariant under $\sigma$. In that case, the Hecke algebra $\mathscr{H}\left(D^{*}, \mathrm{O}^{*}, \epsilon\right)$ has support $D^{*}$ (the support of the Hecke algebra is the union of the supports of its elements). This implies that the Hecke algebra $\mathscr{H}\left(M(F), M_{0}, \epsilon \otimes \epsilon\right)$ has support $M(F)$, and by the Satake isomorphism, that the Hecke algebra $\mathscr{H}(G(F), K, \epsilon$ o det) has support $G(F)$.

Assume now that $\epsilon$ is not invariant under $\sigma$. Then the support of the Hecke algebra $\mathscr{H}\left(D^{*}, \mathcal{O}^{*}, \epsilon\right)$ is the set of elements in $D^{*}$ of even normalized valuation. This implies that the support of $\mathscr{H}\left(M(F), M_{0}, \epsilon \otimes \epsilon\right)$ is the union of the cosets $M_{0} d_{2 a, 2 b}$ for $a, b \in \mathbb{Z}$, and that the support of the Hecke algebra $\mathscr{H}(G(F), K, \epsilon$ odet) is the union of the double cosets $K d_{2 a, 2 b} K$, for $a, b \in \mathbb{Z}$ and $a \geq b$.

For a positive element $s$ in the support of $\mathscr{H}\left(M(F), M_{0}, \epsilon \otimes \epsilon\right)$, let $\tau_{s}$ be the Hecke operator in $\mathscr{H}\left(M(F), M_{0}, \epsilon \otimes \epsilon\right)$ of support $M_{0} s$ and value 1 at $s$, and let $T_{s}$ be the Hecke operator in $\mathscr{H}(G(F), K, \epsilon$ o det) of support $K s K$ and value 1 at $s$.

Claim. $\mathscr{S}^{\prime}\left(T_{s}\right)=\tau_{s}$ for any choice of strictly positive $s \in S(F)$.

Proof. It suffices to prove the claim for $s=d_{2 a, 2 b} \in S(F)$ with $a>b$. We compute $\mathscr{S}^{\prime}\left(T_{s}\right)$ on $d_{2 \alpha, 2 \beta}$ with $\alpha \geq \beta$ in $\mathbb{Z}$,

$$
\varphi^{\prime}\left(T_{s}\right)\left(d_{2 \alpha, 2 \beta}\right)=\sum_{x \in D / O} T_{s}\left(\left(\begin{array}{cc}
1 & x \\
0 & 1
\end{array}\right)\left(\begin{array}{cc}
p_{F}^{\alpha} & 0 \\
0 & p_{F}^{\beta}
\end{array}\right)\right) .
$$

The matrix

$$
\left(\begin{array}{ll}
1 & x \\
0 & 1
\end{array}\right)\left(\begin{array}{cc}
p_{F}^{\alpha} & 0 \\
0 & p_{F}^{\beta}
\end{array}\right)=\left(\begin{array}{cc}
p_{F}^{\alpha} & x p_{F}^{\beta} \\
0 & p_{F}^{\beta}
\end{array}\right)
$$

belongs to $K d_{2 \alpha, 2 \beta} K$ when $x \in \mathcal{O}$.

If $x \notin 0$, then putting $v(x)=-\gamma, \gamma>0$, we have

$$
\left(\begin{array}{cc}
p_{F}^{\alpha} & x p_{F}^{\beta} \\
0 & p_{F}^{\beta}
\end{array}\right)=\left(\begin{array}{cc}
0 & x p_{D}^{\gamma} \\
-x^{-1} p_{D}^{-\gamma} & p_{D}^{\gamma}
\end{array}\right)\left(\begin{array}{cc}
p_{D}^{2 \alpha+\gamma} & 0 \\
0 & p_{D}^{2 \beta-\gamma}
\end{array}\right)\left(\begin{array}{cc}
1 & 0 \\
x^{-1} p_{F}^{\alpha-\beta} & 1
\end{array}\right)
$$

which consequently belongs to $K d_{2 \alpha+\gamma, 2 \beta-\gamma} K$.

If $(2 \alpha, 2 \beta)=(2 a, 2 b)$, we see that only $x \in \mathcal{O}$ contributes to $\mathscr{P}^{\prime}\left(T_{s}\right)\left(d_{2 \alpha, 2 \beta}\right)$ and that this contribution is 1 . Hence $\mathcal{Y}^{\prime}\left(T_{S}\right)\left(d_{2 \alpha, 2 \beta}\right)=1$.

If $(2 \alpha, 2 \beta)=(2 a-\gamma, 2 b+\gamma)$ with $\gamma>0$, we see that the only $x$ contributing to $\mathscr{S}^{\prime}\left(T_{s}\right)\left(d_{2 \alpha, 2 \beta}\right)$ are those with $v(x)=-\gamma$ and that this contribution is 1 . Therefore 
$\mathscr{S}^{\prime}\left(T_{s}\right)\left(d_{2 \alpha, 2 \beta}\right)$ is the number of $x \in D / \mathcal{O}$ of valuation $-\gamma$, and hence

$$
\mathscr{S}^{\prime}\left(T_{S}\right)\left(d_{2 \alpha, 2 \beta}\right)=q^{2 \gamma}-q^{2(\gamma-1)} .
$$

However, $\gamma$ has to be even, so that $\mathscr{S}^{\prime}\left(T_{s}\right)\left(d_{2 \alpha, 2 \beta}\right)=0$.

For the other values of $\alpha, \beta$ in $\mathbb{Z}$, we see that $\mathscr{S}^{\prime}\left(T_{s}\right)\left(d_{2 \alpha, 2 \beta}\right)=0$.

\section{Proof of the main theorem}

We give three lemmas which will help us to study the map $\zeta$ (Proposition 4.9).

Lemma 6.1. The map $\zeta$ is injective on the set of functions $f \in \mathrm{c}-\operatorname{Ind}_{\mathscr{P}}^{G(F)} V_{N(k)}$ with support in $\mathscr{P} Z(F)^{+N} K$.

Proof. Let $f$ be such that $\zeta(f)=0$ with support in $\mathscr{P} Z(F)^{+N} K$. We claim that $f=0$ on $\mathscr{P} P(F)$. This implies that $f=0$, because $G(F)=P(F) K$ and for $k \in K$, the function $k^{-1} f$ satisfies the same conditions as $f$. To prove the claim, we use only that $\zeta(f)(1)=0$ in c-Ind $M_{0}^{M(F)} V_{N(k)}$. As $\zeta(f)(1)$ depends only on the restriction of $f$ to $\mathscr{P} P(F)$ (Remark 4.10), we assume, as we may, that the support of $f$ is contained in $\mathscr{P} P(F)$. The support of $f$ is a finite disjoint union of $\mathscr{P}_{z_{i}} k_{i}$ for $z_{i} \in Z(F)^{+N}$ and $k_{i} \in K$, with $z_{i} k_{i} \in \mathscr{P} P(F)$. We have $\mathscr{P} P(F)=\bar{N}_{0,+} P(F)$, and hence $k_{i} \in z_{i}^{-1} \bar{N}_{0,+} z_{i} P(F)$. As $z_{i}$ is positive, $z_{i}^{-1} \bar{N}_{0,+} z_{i} \subset \bar{N}_{0,+}$. This implies that we can suppose $k_{i} \in P(F) \cap K$. As $P(F) \cap K=N_{0} M_{0}$ and $z_{i}$ is positive, we can suppose $k_{i} \in M_{0}$. We proved that the support of $f$ is a finite disjoint union of $\mathscr{P} z_{i} k_{i}$ for $z_{i} \in Z(F)^{+N}$ and $k_{i} \in M_{0}$. Taking the intersection with $M(F)$, the sets $M(F) \cap \mathscr{P}_{z_{i}} k_{i}$ are also disjoint. Writing

$$
f=\sum_{i}\left(z_{i} k_{i}\right)^{-1}\left[1, \bar{v}_{i}\right]_{\mathscr{P}},
$$

we have $\zeta(f)(1)=\sum_{i}\left(z_{i} k_{i}\right)^{-1}\left[1, \bar{v}_{i}\right]_{M_{0}}$, and $\zeta(f)(1)=0$ is equivalent to $\bar{v}_{i}=0$ for all $i$.

Lemma 6.2. (i) A compact space $P(F) \backslash G(F)$ is given by the $G(F)$-translates of $P(F) \backslash P(F) \bar{N}_{0,+} s^{n}$, for all $n \in \mathbb{N}$.

(ii) For any subset $X \subset G(F)$ with finite image in $\mathscr{P} \backslash G(F)$, there exists a large integer $n \in \mathbb{N}$ such that $s^{n} X \subset \mathscr{P} Z(F)^{+N} K$.

Proof [Herzig 2011, Lemma 2.20]. (i) The compact space $P(F) \backslash G(F)$ is the union of the right $G(F)$-translates of the big cell $P(F) \backslash P(F) \bar{N}(F)$, which is open, and the $s^{-n} \bar{N}_{0,+} s^{n}$ for $n \in \mathbb{N}$ form a decreasing sequence of open subgroups of $\bar{N}(F)$ converging to 1 .

(ii) Let $\mathcal{N}$ be the normalizer of $S$ in $G$ and let $\mathscr{B}$ be the inverse image of $B(k)$ in $K$ (an Iwahori subgroup). Then $(G(F), \mathscr{B}, \mathcal{N}(F))$ is a generalized Tits system [Morris 1993, 3.12]. We have: 
a) $G(F)=\mathscr{B} \mathcal{N}(F) \mathscr{B}$.

b) For $v \in \mathcal{N}(F)$, there is a finite subset $X_{v}$ in $\mathcal{N}(F)$ such that, for all $v^{\prime} \in \mathcal{N}(F)$, we have

$$
v^{\prime} \mathscr{B} v \subset \cup_{x \in X_{v}} \mathscr{B} v^{\prime} x \mathscr{B}
$$

c) As the parahoric group $K$ is special, it contains representatives of the Weyl group, so for any $v \in \mathcal{N}(F)$, there exists $z \in Z(F)$ such that $v K=z K$.

We deduce from a) and c) that $G(F)=\mathscr{B} Z(F) K$. We may assume that $X$ is a finite union $X=\cup_{i} \mathscr{P}_{z_{i}} k_{i}$ with $z_{i} \in Z(F), k_{i} \in K$. We deduce from b) that, for any index $i$, there are finitely many $n_{i, j} \in \mathcal{N}(F)$ such that $z \mathscr{B} z_{i} \subset \cup_{j} \mathscr{B} z n_{i, j} \mathscr{B}$ for all $z \in Z(F)$. It follows that for $n \in \mathbb{N}$ and $z=s^{n}$, we have

$$
z \mathscr{P}_{z_{i}} k_{i} \subset P_{0} z \bar{N}_{0,+} z_{i} k_{i} \subset \cup_{j} \mathscr{P}_{z n_{i, j}} K
$$

as $\bar{N}_{0,+} \subset \mathscr{B}$. We choose $z_{i, j} \in Z(F)$ such that $z_{i, j} K=n_{i, j} K$, as we may by c). There exists $n \in \mathbb{N}$ such that $s^{n} z_{i, j} \in Z(F)^{+N}$ for all $i, j$. Hence $s^{n} X \subset$ $\cup_{j} \mathscr{P} s^{n} z_{i, j} K \subset \mathscr{P} Z(F)^{+N} K$.

Let $\sigma$ be a smooth $C$-representation of $M(F)$. For any nonzero vector $y$ in the space of $\sigma$, there exists a function $f_{y} \in \operatorname{Ind}_{P(F)}^{G(F)} \sigma$ with support $P(F) \bar{N}_{0,+}$ and value $y$ on $\bar{N}_{0+}$ because the multiplication $P(F) \times \bar{N}_{0+} \rightarrow P(F) \bar{N}_{0,+}$ is a homeomorphism.

Lemma 6.3. Let $\sigma$ be a smooth $C$-representation of $M(F)$ generated by an element $x$. Then the representation $\operatorname{Ind}_{P(F)}^{G(F)} \sigma$ is generated by the functions $f_{s^{-n} x}, n \in \mathbb{Z}$, with support $P(F) \bar{N}_{0,+}$ and value $s^{-n} x$ on $\bar{N}_{0+}$.

Proof. By Lemma 6.2, it is enough to show that any function $f_{n, m x} \in \operatorname{Ind}_{P(F)}^{G(F)} \sigma$ with support contained in $P(F) \bar{N}_{0,+} s^{n}$ and value $m x$ on $\bar{N}_{0+} s^{n}$, for $n \in \mathbb{N}$ and $m \in M(F)$, is contained in the subrepresentation generated by $f_{s^{-r} x}$ for all $r \in \mathbb{Z}$. The function $m^{-1} f_{n, m x}$ has support in $P(F) \backslash P(F) \bar{N}_{0+} s^{n} m$ and value $s^{-n} x$ on the compact open subset $m^{-1} s^{-n} \bar{N}_{0+} s^{n} m$ of $\bar{N}(F)$; for some $n^{\prime} \in \mathbb{N}$, this set is a disjoint union of $s^{-n^{\prime}} \bar{N}_{0+} s^{n^{\prime}} \bar{n}$ for $\bar{n}$ running through a finite subset of $\bar{N}(F)$. For a nonzero $y$ in the space of $\sigma$, the function $\left(s^{n^{\prime}} \bar{n}\right)^{-1} f_{y} \in \operatorname{Ind}_{P(F)}^{G(F)} \sigma$ has support $P(F) \bar{N}_{0+} s^{n^{\prime}} \bar{n}$ and value $s^{-n^{\prime}} y$ on $s^{-n^{\prime}} \bar{N}_{0+} s^{n^{\prime}} \bar{n}$. The sum over $\bar{n}$ of $\left(s^{n^{\prime}} \bar{n}\right)^{-1} f_{s^{n^{\prime}-n} x}$ is equal to $m^{-1} f_{n, m x}$.

Proposition 6.4. (i) The image of $\xi$ contains $T_{\mathscr{P}}\left(\mathrm{c}-\operatorname{Ind}_{\mathscr{P}}^{G(F)} V_{N(k)}\right)$ when $V$ is $\bar{P}$-regular.

(ii) The kernel of the map $\zeta$ is the $T_{\mathscr{P}}^{\infty}$-torsion part of $\mathrm{c}-\operatorname{Ind}_{\mathscr{P}}^{G(F)} V_{N(k)}$, and the representation

$$
\mathrm{c}-\operatorname{Ind}_{P(F)}^{G(F)}\left(\mathrm{c}-\operatorname{Ind}_{M_{0}}^{M(F)} V_{N(k)}\right)
$$


is generated by

$$
\left(T_{M}^{-n} \circ \zeta\right)\left([1, \bar{v}]_{\mathscr{P}}\right), \quad \text { for all } n \in \mathbb{Z},
$$

for any fixed nonzero element $\bar{v} \in V_{N(k)}$.

Proof. (i) This follows from Proposition 5.4(i).

(ii) We fix a nonzero $\bar{v} \in V_{N(k)}$; then $x=[1, \bar{v}]_{M_{0}}$ generates the representation $\sigma=\mathrm{c}-\operatorname{Ind}_{M_{0}}^{M(F)} V_{N(k)}$. We note that for $n \in \mathbb{Z}$, by Definition 4.4 and 4.8,

$$
\left(T_{M}^{n} \circ \zeta\right)\left([1, \bar{v}]_{\overline{\mathscr{P}}}\right)=f_{S^{-n} x} .
$$

We obtain by Lemma 6.3 that the representation $\operatorname{Ind}_{P(F)}^{G(F)}\left(\mathrm{c}-\operatorname{Ind}_{M_{0}}^{M(F)} V_{N(k)}\right)$ is generated by the elements $\left(T_{M}^{n} \circ \zeta\right)\left([1, \bar{v}]_{\bar{\Phi}}\right)$, when $n$ runs through $\mathbb{Z}$.

We now consider an element $f$ in the kernel of $\zeta$. The function $f$ vanishes outside of a compact set $X$ with finite image in $\mathscr{P} \backslash G(F)$. We choose an integer $n \in \mathbb{N}$ such that $s^{n} X \subset \mathscr{P} Z(F)^{+N} K$ (Lemma 6.2(ii)). The support of $T_{\mathscr{P}}^{n}$ is $\mathscr{P} s^{n} \mathscr{P}$ by (13) and the positivity of $s$. The support of $T_{\mathscr{P}}^{n}(f)$ is contained in $\mathscr{P} s^{n} X$, and hence in $\mathscr{P} Z(F)^{+N} K$. By Lemma 6.1, we conclude that $T_{\mathscr{P}}^{n}(f)=0$. The converse follows from Proposition 5.4(ii).

In the diagram (12), the representations are $C[T]$-modules, where $T$ acts as on the middle space by $T_{K, \mathscr{P}}$, on the right space by $T_{M}$, and on the left space by $\left(\mathscr{S}^{\prime}\right)^{-1}\left(T_{M}\right)$. Proposition 5.4 tells us that:

- The map $\zeta$ is $C[T]$-linear.

- When $V$ is $\bar{P}$-regular, the map $\xi$ is $C[T]$-linear and $\left(\mathscr{S}^{\prime}\right)^{-1}\left(T_{M}\right)=T_{G}$.

Corollary 6.5. (i) The T-localization $\zeta_{T}$ of $\zeta$ is an isomorphism.

(ii) When $V$ is $\bar{P}$-regular, the $T$-localization $\xi_{T}$ of $\xi$ is an isomorphism.

The map $\Theta$ is the $T$-localization of $I_{V}=\zeta \circ \xi$. By Corollary 6.5(ii), the map $\Theta=\zeta_{T} \circ \xi_{T}$ is surjective when $V$ is $\bar{P}$-regular.

Remark 6.6. We suppose that $V$ is given by a character $\epsilon$ of $K$, and that there exists a character $\epsilon_{M}$ of $M(F)$ equal to $\epsilon$ on $M_{0}$ (such a character $\epsilon_{M}$ does not always exist). We consider the composite of $I_{V}$ with the surjective natural map

$$
\psi: \operatorname{Ind}_{P(F)}^{G(F)}\left(\operatorname{c}^{-\operatorname{Ind}_{M}} M(F) \epsilon\right) \rightarrow \operatorname{Ind}_{P(F)}^{G(F)} \epsilon_{M} .
$$

If $\epsilon_{M}$ extends to a character $\epsilon_{G}$ of $G(F)$, the image of $\psi \circ I_{V}$ is the subrepresentation $\epsilon_{G}$ of dimension 1 of $\operatorname{Ind}_{P(F)}^{G(F)} \epsilon_{M}$, and the map $\psi \circ \Theta$ is nonsurjective.

But in the case where $\epsilon_{M}$ does not extend to a character $\epsilon_{G}$ of $G(F)$, the map $\psi \circ \Theta$ can be surjective. For example, $\psi \circ \Theta$ is surjective when $\operatorname{Ind}_{P(F)}^{G(F)} \epsilon_{M}$ is irreducible. This is the case, for any choice of $\epsilon_{M}$, when $G=U(2,1)$ with respect to an unramified quadratic extension of $F, B$ is a Borel subgroup, and $K$ is a special 
nonhyperspecial parahoric subgroup [Abdellatif 2011]; this is also the case when $G(F)=\mathrm{GL}(2, D)$ with $D$ a quaternion skew field over $F, B$ is the upper triangular subgroup, and $K=\mathrm{GL}\left(2, O_{D}\right)$ [Ly $\left.\geq 2012\right]$.

\section{Supersingular representations of $G(F)$}

We introduce first the notion of $K$-supersingularity for an irreducible smooth representation $\pi$ of $G(F)$. Then we recall the notion of supercuspidality [Henniart and Vigneras 2011, 1.7 footnote]. We expect that supercuspidality is equivalent to $K$-supersingularity, at least for admissible representations. We will give some partial results in this direction. Finally, when $\pi$ is admissible, we give an equivalent definition of $K$-supersingularity which coincides with the definition given by Herzig and Abe when $G$ is $F$-split, $K$ is hyperspecial, and the characteristic of $F$ is 0 .

Let $\pi$ be an irreducible smooth $C$-representation of $G(F)$. For any smooth irreducible $C$-representation $V$ of $K$, we consider

$$
\operatorname{Hom}_{G(F)}\left(\mathrm{c}-\operatorname{Ind}_{K}^{G(F)} V, \pi\right)
$$

as a right module for the Hecke algebra $\mathscr{H}(G(F), K, V)$.

Remark 7.1. Given $\pi$, there exists an irreducible representation $V$ of $K$ such that $\operatorname{Hom}_{G(F)}\left(\mathrm{c}-\operatorname{Ind}_{K}^{G(F)} V, \pi\right) \neq 0$. Indeed, a nonzero element $v \in \pi$ being fixed by an open subgroup of $K$ generates a $K$-stable subspace $W$ of finite dimension; if $V$ is an irreducible subrepresentation of $W$, we have $\operatorname{Hom}_{K}(V, \pi) \neq 0$, and hence the result by Frobenius reciprocity.

For any standard parabolic subgroup $P=M N$, we consider the Satake map

$$
\mathscr{S}^{\prime}=\mathscr{Y}_{M, G}^{\prime}: \mathscr{H}(G(F), K, V) \rightarrow \mathscr{H}\left(M(F), M_{0}, V_{N(k)}\right) .
$$

We recall that $\mathscr{Y}^{\prime}$ is a localization at some element $T_{M}$ (Proposition 4.5).

Definition 7.2. An irreducible smooth $C$-representation $\pi$ of $G(F)$ is called $K$ supersingular when

$$
\mathscr{H}\left(M(F), M_{0}, V_{N(k)}\right) \otimes \mathscr{H}(G(F), K, V), \mathscr{S}^{\prime} \operatorname{Hom}_{G(F)}\left(\mathrm{c}-\operatorname{Ind}_{K}^{G(F)} V, \pi\right)=0,
$$

for all irreducible smooth $C$-representations $V$ of $K$ and all standard Levi subgroups $M \neq G$.

The condition means that the localization of the right $\mathscr{H}(G(F), K, V)$-module

$$
\operatorname{Hom}_{G(F)}\left(\mathrm{c}-\operatorname{Ind}_{K}^{G(F)} V, \pi\right)
$$

at $T_{M}$ is 0 , that is, for any nonzero $f \in \operatorname{Hom}_{G(F)}\left(\mathrm{c}-\operatorname{Ind}_{K}^{G(F)} V, \pi\right)$, there is $n \in \mathbb{N}$ such that $\mathscr{S}^{\prime-1}\left(T_{M}^{n}\right)(f)=0$. If the space $\operatorname{Hom}_{G(F)}\left(\mathrm{c}-\operatorname{Ind}_{K}^{G(F)} V, \pi\right)$ is finite-dimensional, this means that the eigenvalues of $\mathscr{G}^{\prime-1}\left(T_{M}\right)$ on this space are 0 , or equivalently, 
that the characters of $\mathscr{L}(G(F), K, V)$ appearing in

$$
\operatorname{Hom}_{G(F)}\left(\mathrm{c}-\operatorname{Ind}_{K}^{G(F)} V, \pi\right)
$$

vanish at $\mathscr{Y}^{\prime-1}\left(T_{M}\right)$. For admissible representations, our definition is equivalent to the one given by Herzig [2011, Definition 4.7] and Abe [2011, Definition 5.1].

Definition 7.3. An irreducible smooth $C$-representation $\pi$ of $G(F)$ is called supercuspidal if $\pi$ is not isomorphic to a subquotient of $\mathrm{c}-\operatorname{Ind}_{P(F)}^{G(F)} \tau$ for a proper standard parabolic subgroup $P=M N$ of $G$ and for an irreducible smooth $C$-representation $\tau$ of $M(F)$.

The definition, which is valid for any field $C$, does not depend on the minimal parabolic $F$-subgroup $B$ of $G$ used to define the standard parabolic subgroups, as all such $B$ 's are conjugate in $G(F)$. Consequently, we get an equivalent definition if we let $P$ be any parabolic subgroup different from $G$.

Let $V$ be an irreducible smooth $C$-representation of $K$, let $P=M N$ be a proper standard parabolic subgroup of $G$, and let $\sigma$ be a smooth $C$-representation of $M(F)$. Our first result concerns the $T_{M}$-localization of the right $\mathscr{H}(G(F), K, V)$-module

$$
\operatorname{Hom}_{G(F)}\left(\mathrm{c}-\operatorname{Ind}_{K}^{G(F)} V, \operatorname{Ind}_{P(F)}^{G(F)} \sigma\right) .
$$

Proposition 7.4. (i) $\left.V \subset\left(\operatorname{Ind}_{P(F)}^{G(F)} \sigma\right)\right|_{K}$ if and only if $\left.V_{N(k)} \subset \sigma\right|_{M_{0}}$.

(ii) In this case, the action of $\mathscr{Y}^{\prime-1}\left(T_{M}\right)$ on $\operatorname{Hom}_{G(F)}\left(\mathrm{c}-\operatorname{Ind}_{K}^{G(F)} V, \operatorname{Ind}_{P(F)}^{G(F)} \sigma\right)$ is invertible.

Proof. (See [Herzig 2011, p. 416].) (i) This follows from the isomorphism (2).

(ii) By (4), we have isomorphisms of $\mathscr{H}(G(F), K, V)$-modules

$$
\operatorname{Hom}_{G(F)}\left(\operatorname{c-Ind}_{K}^{G(F)} V, \operatorname{Ind}_{P(F)}^{G(F)} \sigma\right) \simeq \operatorname{Hom}_{K}\left(V, \operatorname{Ind}_{P(F)}^{G(F)} \sigma\right) \simeq \operatorname{Hom}_{M_{0}}\left(V_{N(k)}, \sigma\right),
$$

where $\mathscr{H}(G(F), K, V)$ acts on the final term by $\mathscr{S}^{\prime}$; the last isomorphism follows from Frobenius reciprocity and $K \cap P(F)=(K \cap M(F))(K \cap N(F))$. The claim follows since $\mathscr{S}^{\prime}$ is a localization map at $T_{M}$, by Proposition 4.5.

Our results on the comparison between non- $K$-supersingular and nonsupercuspidal irreducible smooth $C$-representations of $G(F)$ are:

Proposition 7.5. Let $\tau$ be an irreducible smooth $C$-representation of $M(F)$.

(i) An irreducible subrepresentation of $\operatorname{Ind}_{P(F)}^{G(F)} \tau$ is not $K$-supersingular.

(ii) An admissible irreducible quotient of $\operatorname{Ind}_{P(F)}^{G(F)} \tau$ is not $K$-supersingular. 
This proposition claims that certain nonsupercuspidal irreducible representations of $G(F)$ are non- $K$-supersingular. The next proposition claims that certain non- $K$ supersingular admissible irreducible representations of $G(F)$ are nonsupercuspidal.

Proposition 7.6. Let $\pi$ be an admissible irreducible smooth $C$-representation of $G(F)$, let $P=M N \subset Q=L N^{\prime}$ be two standard parabolic $F$-subgroups different from $G$, and let $V$ be a $\bar{Q}$-regular irreducible smooth $C$-representation of $K$ such that the localization of the right $\mathscr{H}(G(F), K, V)$-module

$$
\operatorname{Hom}_{G(F)}\left(\mathrm{c}-\operatorname{Ind}_{K}^{G(F)} V, \pi\right)
$$

at $T_{M}$ is not 0. Then $\pi$ is a quotient of $\operatorname{Ind}_{Q(F)}^{G(F)} \tau$ for an admissible irreducible smooth $C$-representation $\tau$ of $L(F)$.

Proof of Proposition 7.5. (i) Proposition 7.4 implies that an irreducible subrepresentation of $\operatorname{Ind}_{P(F)}^{G(F)} \tau$ is not $K$-supersingular.

(ii) Let $\pi$ be an irreducible quotient of $\operatorname{Ind}_{P(F)}^{G(F)} \tau$. We choose an irreducible smooth $C$-representation $W$ of $M_{0}$ such that the irreducible representation $\tau$ is a quotient of $\mathrm{c}-\operatorname{Ind}_{M_{0}}^{M(F)} W$. Then $\pi$ is a quotient of $\operatorname{Ind}_{P(F)}^{G(F)}\left(\mathrm{c}-\operatorname{Ind}_{M_{0}}^{M(F)} W\right)$. We consider the unique irreducible $\bar{P}$-regular representation $V$ of $G(k)$ such that $V_{N(k)} \simeq W$ (Proposition 3.10). By our main theorem (Theorem 4.6),

$$
\operatorname{Ind}_{P(F)}^{G(F)}\left(\mathrm{c}-\operatorname{Ind}_{M(F) \cap K}^{M(F)} W\right) \simeq \mathscr{H}\left(M(F), M_{0}, V_{N(k)}\right) \otimes_{\mathcal{H}(G(F), K, V), \mathscr{S}^{\prime}} \mathrm{c}-\operatorname{Ind}_{K}^{G(F)} V .
$$

We deduce:

$$
\operatorname{Hom}_{G(F)}\left(\mathscr{H}\left(M(F), M_{0}, V_{N(k)}\right) \otimes_{\mathscr{H}(G(F), K, V), \mathscr{S}^{\prime}} \mathrm{c}-\operatorname{Ind}_{K}^{G(F)} V, \pi\right) \neq 0 .
$$

If $\pi$ is admissible, we will show

$$
\mathscr{H}\left(M(F), M_{0}, V_{N(k)}\right) \otimes \mathscr{H}(G(F), K, V), \mathscr{S}^{\prime} \operatorname{Hom}_{G(F)}\left(\mathrm{c}-\operatorname{Ind}_{K}^{G(F)} V, \pi\right) \neq 0 .
$$

This implies that $\pi$ is not $K$-supersingular.

To prove (22), we write $X:={\mathrm{c}-\operatorname{Ind}_{K}^{G(F)}}^{(F)} T:=T_{M} \in A:=\mathscr{H}(G(F), K, V)$, $B=A\left[T^{-1}\right]$. Our assumption is

$$
\operatorname{Hom}_{G}\left(B \otimes_{A} X, \pi\right) \neq 0,
$$

and we want to prove that $B \otimes_{A} \operatorname{Hom}_{G}(X, \pi) \neq 0$, provided that $\operatorname{Hom}_{G}(X, \pi)$ is finite-dimensional (which is the case if $\pi$ is admissible).

We consider the natural linear map

$$
r: \operatorname{Hom}_{G}\left(B \otimes_{A} X, \pi\right) \rightarrow \operatorname{Hom}_{G}(X, \pi), \quad \varphi \mapsto(x \mapsto \varphi(1 \otimes x)) .
$$


The space $\operatorname{Hom}_{G}\left(B \otimes_{A} X, \pi\right)$ is naturally a right $B$-module, and hence a right $A$-module by restriction. The map $r$ is $A$-linear:

$$
r(\varphi a)(x)=(\varphi a)(1 \otimes x)=\varphi(a \otimes x)=\varphi(1 \otimes a x)=r(\varphi)(a x)=(r(\varphi) a)(x),
$$

for $a \in A, x \in X, \varphi \in \operatorname{Hom}_{G}\left(B \otimes_{A} X, \pi\right)$. Consequently, the image $\operatorname{Im}(r)$ is an $A$-submodule of $\operatorname{Hom}_{G}(X, \pi)$. We remark that $\operatorname{Im}(r) T=\operatorname{Im}(r)$ because $r(\varphi)=$ $r\left(\varphi T^{-1}\right) T$ for $\varphi \in \operatorname{Hom}_{G}\left(B \otimes_{A} X, \pi\right)$.

We show now that our hypothesis implies that $\operatorname{Im}(r)$ is not 0 . Indeed, let $\varphi \neq 0$ in $\operatorname{Hom}_{G}\left(B \otimes_{A} X, \pi\right)$. There exist $b \in B$ and $x \in X$ such that $\varphi(b \otimes x) \neq 0$. Writing $b=T^{-n} a$ with $n \in \mathbb{N}$ and $a \in A$, we get $\varphi\left(T^{-n} a \otimes x\right)=\varphi T^{-n}(1 \otimes a x) \neq 0$ so that $r\left(\varphi T^{-n}\right) \neq 0$.

Assume now that $\operatorname{Hom}_{G}(X, \pi)$ is finite-dimensional. Then $\operatorname{Im}(r)$ is also finitedimensional and as $\operatorname{Im}(r) T=\operatorname{Im}(r), T$ induces an automorphism of $\operatorname{Im}(r)$ so that $B \otimes_{A} \operatorname{Im}(r) \neq 0$. The localization being an exact functor, we have

$$
B \otimes_{A} \operatorname{Hom}_{G}(X, \pi) \neq 0 .
$$

We state a useful general lemma before proving Proposition 7.6.

Let $R$ be a commutative ring, let $\mathscr{H}$ be an $R$-algebra, let $W$ be a left $\mathscr{H}$-module with a smooth $\mathscr{H}$-linear action of $M(F)$, and let $\mathcal{N}$ be a right $\mathscr{H}$-module. Then $\mathcal{N} \otimes_{\mathscr{H}} W$ is a smooth $R$-representation of $M(F)$ and we can form $\operatorname{Ind}_{P(F)}^{G(F)}\left(\mathcal{N} \otimes_{\mathcal{H}} W\right)$. We can also form $\mathcal{N} \otimes \mathscr{H} \operatorname{Ind}_{P(F)}^{G(F)}(W)$, where the structure of left $\mathscr{H}$-module on $\operatorname{Ind}_{P(F)}^{G(F)}(W)$ is given by $(h, f) \rightarrow h f: g \rightarrow h(f(g))$. The canonical map

$$
\iota_{\mathcal{N}}: \mathcal{N} \otimes_{\mathscr{H}} \operatorname{Ind}_{P(F)}^{G(F)}(W) \rightarrow \operatorname{Ind}_{P(F)}^{G(F)}\left(\mathcal{N} \otimes_{\mathscr{H}} W\right)
$$

is clearly $G(F)$-equivariant.

Lemma 7.7. The map $\iota_{\mathcal{N}}$ is an isomorphism.

Proof. It is well known that the quotient map $G(F) \rightarrow P(F) \backslash G(F)$ admits a continuous section and that the module $C^{\infty}(P(F) \backslash G(F), R)$ is free. This implies that the parabolic induction functor $\operatorname{Ind}_{P(F)}^{G(F)}$ - for smooth $R$-representations is exact and commutes with infinite direct sums, and that $\operatorname{Ind}_{P(F)}^{G(F)}(W)$ identifies with $C^{\infty}(G(F) / P(F), R) \otimes W$ as $R$-modules, for any smooth $R$-representation $W$ of $M(F)$.

We choose a resolution of $\mathcal{N}$ by free right $\mathscr{H}$-modules

$$
\mathscr{F}_{1} \rightarrow \mathscr{F}_{0} \rightarrow \mathcal{N} \rightarrow 0 \text {. }
$$

We have a commutative diagram 


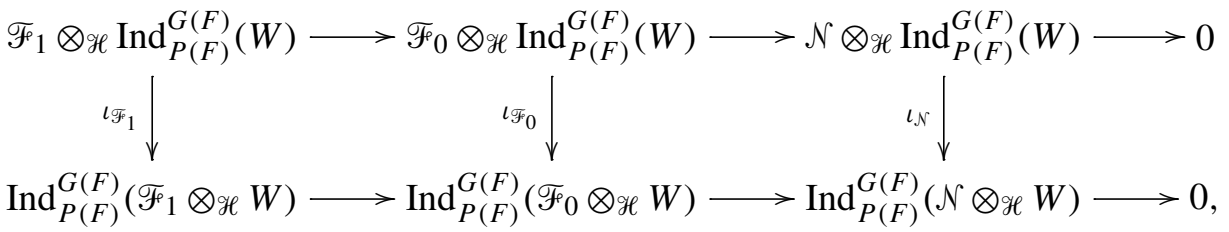

where the lines are exact, the second one because $\operatorname{Ind}_{P(F)}^{G(F)}$ is an exact functor. The functor Ind $\operatorname{In}_{P(F)}^{G(F)}$ being compatible with direct sums, the maps $\iota_{\mathscr{F}_{1}}$ and $\iota \mathscr{F}_{0}$ are isomorphisms. It follows that $\iota_{\mathcal{N}}$ is an isomorphism.

Remark 7.8. When $\pi$ is an admissible smooth $C$-representation of $G$, then

$$
\operatorname{Hom}_{G(F)}\left(\mathrm{c}-\operatorname{Ind}_{K}^{G(F)} V, \pi\right)
$$

is finite-dimensional, and hence it is 0 or contains a simple $\mathscr{H}(G(F), K, V)$-module.

An irreducible smooth $C$-representation $\pi$ of $G(F)$ such that

$$
\operatorname{Hom}_{G(F)}\left(\mathrm{c}-\operatorname{Ind}_{K}^{G(F)} V, \pi\right)
$$

contains a simple $\mathscr{H}(G(F), K, V)$-module $\mathcal{N}$ has a central character. This follows from:

1. The center of $\mathscr{H}(G(F), K, V)$ acts on $\mathcal{N}$ by a character [Vigneras 2007].

2. $\pi$ is a quotient of $\mathcal{N} \otimes \mathscr{H}(G(F), K, V) \mathrm{c}-\operatorname{Ind}_{K}^{G(F)} V$.

Proof of Proposition 7.6. Put

$$
\mathscr{H}_{L, V, \pi}:=\mathscr{H}\left(L(F), L_{0}, V_{N^{\prime}(k)}\right) \otimes_{\mathscr{H}(G(F), K, V), \mathscr{S}_{L, G}^{\prime}} \operatorname{Hom}_{G(F)}\left(\mathrm{c}-\operatorname{Ind}_{K}^{G(F)} V, \pi\right),
$$

and similarly define $\mathscr{H}_{M, V, \pi}$. From the transitivity $\mathscr{Y}_{M, G}^{\prime}=\mathscr{Y}_{M, L}^{\prime} \circ \mathscr{Y}_{L, G}^{\prime}$, we deduce

$$
\mathscr{H}_{M, V, \pi}=\mathscr{H}\left(M(F), M_{0}, V_{N(k)}\right) \otimes_{\mathscr{H}\left(L(F), L_{0}, V_{N^{\prime}(k)}\right), \mathscr{S}_{M, L}^{\prime}} \mathscr{H}_{L, V, \pi} .
$$

Hence $\mathscr{H}_{L, V, \pi}$ is not 0 , because $\mathscr{H}_{M, V, \pi} \neq 0$. The space

$$
\operatorname{Hom}_{G(F)}\left(\mathrm{c}-\operatorname{Ind}_{K}^{G(F)} V, \pi\right)
$$

is finite-dimensional because $\pi$ is admissible, and we have just seen that its localization at $T_{L}$ is not 0 . Therefore $T_{L}$ has a nonzero eigenvalue $\alpha$. The corresponding eigenspace is a nonzero $\mathscr{H}(G(F), K, V)$-submodule, and hence contains a simple right $\mathscr{H}(G(F), K, V)$-submodule $\mathcal{N}$, which we consider as a simple $\mathscr{H}\left(L(F), L_{0}, V_{N^{\prime}(k)}\right)$-module with $T_{L}$ acting by $\alpha$. The irreducible representation $\pi$ is a quotient of

$$
\mathcal{N} \otimes \mathscr{H}(G(F), K, V) \mathrm{c}-\operatorname{Ind}_{K}^{G(F)} V .
$$


As $V$ is $\bar{Q}$-regular, the representation (23) is isomorphic to

$$
\mathcal{N} \otimes \mathscr{H}_{\left(L(F), L_{0}, V_{N^{\prime}(k)}\right)} \operatorname{Ind}_{Q(F)}^{G(F)}\left(\mathrm{c}-\operatorname{Ind}_{L_{0}}^{L(F)} V_{N^{\prime}(k)}\right)
$$

by Theorem 4.6. By Lemma 7.7, this last representation is isomorphic to $\operatorname{Ind}_{Q(F)}^{G(F)} \sigma$, where

$$
\sigma:=\mathcal{N} \otimes \mathscr{H}\left(L(F), L_{0}, V_{N^{\prime}(k)}\right) \mathrm{c}-\operatorname{Ind}_{L_{0}}^{L(F)} V_{N^{\prime}(k)}
$$

is a smooth representation of $L(F)$. The center of $L(F)$ embeds naturally in the center of the Hecke algebra $\mathscr{H}\left(L(F), L_{0}, V_{N^{\prime}(k)}\right)$ and acts by a character on the simple $\mathscr{H}\left(L(F), L_{0}, V_{N^{\prime}(k)}\right)$-module $\mathcal{N}$. Hence $\sigma$ has a central character.

The admissible irreducible representation $\pi$ is a quotient of $\operatorname{Ind}_{Q(F)}^{G(F)} \sigma$, where $\sigma$ has a central character. By Proposition 7.9 below, $\pi$ is a quotient of $\operatorname{Ind}_{Q(F)}^{G(F)} \tau$ for an admissible irreducible smooth $C$-representation $\tau$ of $L(F)$.

Proposition 7.9. Let $\pi$ be an admissible irreducible smooth $C$-representation of $G(F)$ which is a quotient of $\operatorname{Ind}_{P(F)}^{G(F)} \sigma$ for a smooth $C$-representation $\sigma$ of $M(F)$ with a central character. Then there exists an admissible irreducible smooth $C$ representation $\tau$ of $M(F)$ such that $\pi$ is a quotient of $\operatorname{Ind}_{P(F)}^{G(F)} \tau$.

When the characteristic of $F$ is 0, Herzig [2011, Lemma 9.9] proved this proposition using the $\bar{P}$-ordinary functor $\operatorname{Ord}_{\bar{P}}$ introduced by Emerton [2010]. His proof contains four steps:

1. As $\sigma$ is locally $Z_{M}$-finite, we have

$$
\operatorname{Hom}\left(\operatorname{Ind}_{P(F)}^{G(F)} \sigma, \pi\right) \simeq \operatorname{Hom}_{M(F)}\left(\sigma, \operatorname{Ord}_{\bar{P}} \pi\right) .
$$

2. As $\pi$ is admissible, $\operatorname{Ord}_{\bar{P}} \pi$ is admissible.

3. As $\operatorname{Ord}_{\bar{P}} \pi$ is admissible and nonzero, it contains an admissible irreducible subrepresentation $\tau$.

4. As $\operatorname{Ord}_{\bar{P}}$ is the right adjoint of $\operatorname{Ind}_{P(F)}^{G(F)}$ in the category of admissible representations, $\pi$ is a quotient of $\operatorname{Ind}_{P(F)}^{G(F)} \tau$.

The proof is valid without hypothesis on the characteristic of $F$ : we checked carefully that Emerton's proof of steps 1, 2, 4 never uses the characteristic of $F$. Only the proof of step 3 given by Herzig has to be replaced by a characteristic-free proof.

Lemma 7.10. A nonzero admissible smooth $C$-representation of $G(F)$ contains an admissible irreducible subrepresentation.

Proof. Let $\pi$ be a nonzero admissible smooth $C$-representation of $G(F)$, and $H$ an open pro- $p$-subgroup of $G(F)$. The dimension of $\pi^{H}$ is a positive integer. Choose a subrepresentation $\pi_{1}$ of $\pi$ such that $\pi_{1}^{H}$ has minimal positive dimension; then the subrepresentation generated by $\pi_{1}^{H}$ is irreducible. 
This ends the proof Proposition 7.9, and hence of Proposition 7.6.

\section{Acknowledgements}

We would like to thank Noriyuki Abe for his helpful remarks which in particular saved us from a blunder in Example 5.6. We also thank the excellent referee for his care and rapidity.

\section{References}

[Abdellatif 2011] R. Abdellatif, Autour des représentations modulo p des groupes réductifs p-adiques de rang 1, thesis, Faculté des sciences d'Orsay, 2011, www.math.u-psud.fr/ abdellat/RAThese.pdf.

[Abe 2011] N. Abe, "On a classification of admissible irreducible modulo $p$ representations of a p-adic split reductive group”, preprint, 2011.

[Barthel and Livné 1994] L. Barthel and R. Livné, "Irreducible modular representations of $\mathrm{GL}_{2}$ of a local field”, Duke Math. J. 75:2 (1994), 261-292. MR 95g:22030 Zbl 0826.22019

[Borel and Tits 1965] A. Borel and J. Tits, "Groupes réductifs", Inst. Hautes Études Sci. Publ. Math. 27 (1965), 55-150. MR 34 \#7527 Zbl 0145.17402

[Bruhat and Tits 1984] F. Bruhat and J. Tits, "Groupes réductifs sur un corps local, II: Schémas en groupes. Existence d'une donnée radicielle valuée”, Inst. Hautes Études Sci. Publ. Math. 60 (1984), 197-376. MR 86c:20042 Zbl 0597.14041

[Cabanes and Enguehard 2004] M. Cabanes and M. Enguehard, Representation theory of finite reductive groups, New Mathematical Monographs 1, Cambridge University Press, 2004. MR 2005g: 20067 Zbl 1069.20032

[Curtis 1970] C. W. Curtis, "Modular representations of finite groups with split $(B, N)$-pairs", pp. 57-95 in Seminar on Algebraic Groups and Related Finite Groups (Princeton, 1968/69), edited by A. Borel, Springer, Berlin, 1970. MR 41 \#6991 Zbl 0233.20005

[Emerton 2010] M. Emerton, "Ordinary parts of admissible representations of $p$-adic reductive groups, I: Definition and first properties”, Astérisque 331 (2010), 355-402. MR 2011k:22013 Zbl 1205.22013

[Henniart and Vigneras 2011] G. Henniart and M.-F. Vigneras, "A Satake isomorphism for representations modulo $p$ of reductive groups over local fields", preprint, 2011, www.math.jussieu.fr/ $\sim$ vigneras/satake_isomorphism-1.pdf.

[Herzig 2009] F. Herzig, "The weight in a Serre-type conjecture for tame $n$-dimensional Galois representations", Duke Math. J. 149:1 (2009), 37-116. MR 2010f:11083 Zbl 1232.11065

[Herzig 2011] F. Herzig, "The classification of irreducible admissible mod $p$ representations of a p-adic GLn”, Invent. Math. 186:2 (2011), 373-434. MR 2845621 Zbl 1235.22030

$[\mathrm{Ly} \geq 2012] \mathrm{T}$. Ly, "Irreducible representations modulo $p$ representations of $\mathrm{GL}(2, D)$ ", preprint forthcoming.

[Morris 1993] L. Morris, “Tamely ramified intertwining algebras", Invent. Math. 114:1 (1993), 1-54. MR 94g:22035 Zbl 0854.22022

[Vigneras 2007] M.-F. Vigneras, "Représentations irréductibles de GL(2, F) modulo p", pp. 548-563 in L-functions and Galois representations, edited by D. Burns et al., London Math. Soc. Lecture Note Ser. 320, Cambridge Univ. Press, 2007. MR 2009h:11084 Zbl 1172.11017 
Received July 17, 2012. Revised October 6, 2012.

GuY HENNIART

LABORATOIRE DE MATHÉMATIQUES D'ORSAY

UNIVERSITÉ PARIS-SUD

CNRS, UMR 8628, ORSAY CEDEX F-91405

FRANCE

guy.henniart@math.u-psud.fr

Marie-France Vigneras

Institut de MAThÉmatiQues de JUSSiEU

UNIVERSITÉ DE PARIS 7

175 RUE DU CHEVALERET

PARIS 75013

FRANCE

vigneras@math.jussieu.fr 


\title{
PACIFIC JOURNAL OF MATHEMATICS
}

\author{
http://pacificmath.org
}

Founded in 1951 by E. F. Beckenbach (1906-1982) and F. Wolf (1904-1989)

\section{EDITORS}

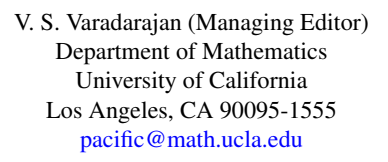

Don Blasius

Department of Mathematics University of California

Los Angeles, CA 90095-1555

blasius@math.ucla.edu

Robert Finn

Department of Mathematics

Stanford University

Stanford, CA 94305-2125

finn@math.stanford.edu

Alexander Merkurjev

Department of Mathematics

University of California

Los Angeles, CA 90095-1555

merkurev@math.ucla.edu

\author{
Vyjayanthi Chari \\ Department of Mathematics \\ University of California \\ Riverside, CA 92521-0135 \\ chari@math.ucr.edu \\ Kefeng Liu \\ Department of Mathematics \\ University of California \\ Los Angeles, CA 90095-1555 \\ liu@math.ucla.edu \\ Sorin Popa \\ Department of Mathematics \\ University of California \\ Los Angeles, CA 90095-1555 \\ popa@math.ucla.edu \\ Paul Yang \\ Department of Mathematics \\ Princeton University \\ Princeton NJ 08544-1000 \\ yang@math.princeton.edu
}

\section{PRODUCTION}

Silvio Levy, Scientific Editor, pacific@math.berkeley.edu

\section{SUPPORTING INSTITUTIONS}

ACADEMIA SINICA, TAIPEI

CALIFORNIA INST. OF TECHNOLOGY

INST. DE MATEMÁTICA PURA E APLICADA

KEIO UNIVERSITY

MATH. SCIENCES RESEARCH INSTITUTE

NEW MEXICO STATE UNIV.

OREGON STATE UNIV.

\author{
STANFORD UNIVERSITY \\ UNIV. OF BRITISH COLUMBIA \\ UNIV. OF CALIFORNIA, BERKELEY \\ UNIV. OF CALIFORNIA, DAVIS \\ UNIV. OF CALIFORNIA, LOS ANGELES \\ UNIV. OF CALIFORNIA, RIVERSIDE \\ UNIV. OF CALIFORNIA, SAN DIEGO \\ UNIV. OF CALIF., SANTA BARBARA
}

\author{
Daryl Cooper \\ Department of Mathematics \\ University of California \\ Santa Barbara, CA 93106-3080 \\ cooper@math.ucsb.edu \\ Jiang-Hua Lu \\ Department of Mathematics \\ The University of Hong Kong \\ Pokfulam Rd., Hong Kong \\ jhlu@maths.hku.hk
}

\section{Jie Qing}

Department of Mathematics

University of California

Santa Cruz, CA 95064

qing@cats.ucsc.edu

These supporting institutions contribute to the cost of publication of this Journal, but they are not owners or publishers and have no responsibility for its contents or policies.

See inside back cover or pacificmath.org for submission instructions.

The subscription price for 2012 is US \$420/year for the electronic version, and \$485/year for print and electronic.

Subscriptions, requests for back issues from the last three years and changes of subscribers address should be sent to Pacific Journal of Mathematics, P.O. Box 4163, Berkeley, CA 94704-0163, U.S.A. Prior back issues are obtainable from Periodicals Service Company, 11 Main Street, Germantown, NY 12526-5635. The Pacific Journal of Mathematics is indexed by Mathematical Reviews, Zentralblatt MATH, PASCAL CNRS Index, Referativnyi Zhurnal, Current Mathematical Publications and the Science Citation Index.

The Pacific Journal of Mathematics (ISSN 0030-8730) at the University of California, c/o Department of Mathematics, 969 Evans Hall, Berkeley, CA 94720-3840, is published monthly except July and August. Periodical rate postage paid at Berkeley, CA 94704, and additional mailing offices. POSTMASTER: send address changes to Pacific Journal of Mathematics, P.O. Box 4163, Berkeley, CA 94704-0163.

PJM peer review and production are managed by EditFlow ${ }^{\circledR}$ from Mathematical Sciences Publishers.

\section{PUBLISHED BY}

\section{mathematical sciences publishers}

http://msp.org/

A NON-PROFIT CORPORATION

Typeset in LATEX 


\section{PACIFIC JOURNAL OF MATHEMATICS}

Volume $260 \quad$ No. $2 \quad$ December 2012

\section{Special issue \\ devoted to the memory of Jonathan Rogawski}

In memoriam: Jonathan Rogawski

257

DON BLASIUS, DINAKAR RAMAKRISHNAN and V. S. VARADARAJAN

$p$-adic Rankin $L$-series and rational points on CM elliptic curves

261

Massimo Bertolini, Henri DARMON and KARTIK PRASANNA

The syntomic regulator for $K_{4}$ of curves

AMNON BESSER and ROB DE JEU

Unique functionals and representations of Hecke algebras

381

BENJAMIN BRUBAKER, DANIEL BUMP and SOLOMON FRIEDBERG

A relative trace formula for PGL(2) in the local setting

395

BROOKE FEIGON

On the degrees of matrix coefficients of intertwining operators

433

TOBIAS FINIS, EREZ LAPID and WERNER MÜLlER

Comparison of compact induction with parabolic induction

457

Guy HENNIART and MARIE-FranCE Vigneras

The functional equation and beyond endoscopy

497

P. EDWARD HERMAN

A correction to Conducteur des Représentations du groupe linéaire

HERVÉ JACQUET

Modular $L$-values of cubic level

ANDREW KNIGHTLY and CHARLES LI

On occult period maps

STEPHEN KUDLA and MiCHAEL RAPOPORT

A prologue to "Functoriality and reciprocity", part I

ROBERT LANGLANDS

Truncation of Eisenstein series

EREZ LAPID and KeITH OUELLETTE

Some comments on Weyl's complete reducibility theorem

JONATHAN ROGAWSKI and V. S. VARADARAJAN

On equality of arithmetic and analytic factors through local Langlands correspondence

FREYDOON SHAHIDI 